\title{
Heterogeneous Nuclear Ribonucleoprotein K, an RNA-Binding Protein, Is Required for Optic Axon Regeneration in Xenopus laevis
}

\author{
Yuanyuan Liu, ${ }^{1 \star}$ Hurong Yu, ${ }^{1,2 *}$ Sarah K. Deaton, ${ }^{1}$ and Ben G. Szaro ${ }^{1}$ \\ ${ }^{1}$ Department of Biological Sciences and the Center for Neuroscience Research, University at Albany, State University of New York, Albany, NY 12222, and \\ 2Department of Anatomy and Histoembryology, Shanghai Medical College, Fudan University, Shanghai 200032, People's Republic of China
}

\begin{abstract}
Axotomized optic axons of Xenopus laevis, in contrast to those of mammals, retain their ability to regenerate throughout life. To better understand the molecular basis for this successful regeneration, we focused on the role of an RNA-binding protein, heterogeneous nuclear ribonucleoprotein (hnRNP) K, because it is required for axonogenesis during development and because several of its RNA targets are under strong post-transcriptional control during regeneration. At $11 \mathrm{~d}$ after optic nerve crush, hnRNP K underwent significant translocation into the nucleus of retinal ganglion cells (RGCs), indicating that the protein became activated during regeneration. To suppress its expression, we intravitreously injected an antisense Vivo-Morpholino oligonucleotide targeting hnRNP K. In uninjured eyes, it efficiently knocked down hnRNP K expression in only the RGCs, without inducing either an axotomy response or axon degeneration. After optic nerve crush, staining for multiple markers of regenerating axons showed no regrowth of axons beyond the lesion site with hnRNP K knockdown. RGCs nonetheless responded to the injury by increasing expression of multiple growthassociated RNAs and experienced no additional neurodegeneration above that normally seen with optic nerve injury. At the molecular level, hnRNP K knockdown during regeneration inhibited protein, but not mRNA, expression of several known hnRNP K RNA targets (NF-M, GAP-43) by compromising their efficient nuclear transport and disrupting their loading onto polysomes for translation. Our study therefore provides evidence of a novel post-transcriptional regulatory pathway orchestrated by hnRNP K that is essential for successful CNS axon regeneration.
\end{abstract}

\section{Introduction}

In Xenopus frogs and other anamniotes, crushed optic axons successfully regenerate functional connections throughout life, whereas in amniotes, regeneration ultimately fails (Sperry, 1944, 1948; Gaze, 1959; Taylor et al., 1989; Tanaka and Ferretti, 2009). Successful regeneration is marked by the execution of a growthassociated program of changes in gene expression (Benowitz et al., 1981; Skene and Willard, 1981; Glasgow et al., 1992; Gervasi et al., 2003). Two of these genes, GAP-43 and the medium neurofilament $N F-M$, directly play roles in facilitating regeneration. Suppressing GAP-43 expression in vivo induces errors in pathfinding, whereas increasing it promotes axonal outgrowth and

Received Oct. 14, 2011; revised Jan. 17, 2012; accepted Jan. 19, 2012.

Author contributions: Y.L., H.Y., and B.G.S. designed research; Y.L., H.Y., S.K.D., and B.G.S. performed research; Y.L., H.Y., and B.G.S. analyzed data; Y.L. and B.G.S. wrote the paper.

Financial support came from the National Science Foundation (IOS 951043) and an American Heart Association predoctoral fellowship (Y.L.). We thank Drs. Itzhak Fischer (Drexel University) and Beatrice Cardinali (Istituto di Biologia (ellulare, Italy) for tau and Xenopus hnRNP E2 antibodies, respectively. We also thank Dr. Christine Gervasi for help with spawning and rearing Xenopus, Dr. Min-Ho Lee for providing access to critical equipment for polysomal profiling, Dr. Kurt M. Gibbs for providing sections of spinal cord-transected tadpole, and Gregory M. Szczepanski for his help with pilot experiments testing the effectiveness of morpholino injection. Chen Wang and Erica Hutchins made helpful editorial comments on this manuscript.

*Y.L. and H.Y. contributed equally to this work.

Correspondence should be addressed to Ben G. Szaro, Department of Biological Sciences, University at Albany, SUNY, 1400 Washington Avenue, Albany, NY 12222. E-mail: bgs86@albany.edu.

DOI:10.1523/JNEUROSCI.5197-11.2012

Copyright $\odot 2012$ the authors $\quad 0270-6474 / 12 / 323563-12 \$ 15.00 / 0$ enhances arborization (Strittmatter et al., 1995; Kruger et al., 1998; Bomze et al., 2001; Zhang et al., 2005; Leu et al., 2010). Meanwhile, removing neurofilaments reduces rates of axon elongation in both developing and regenerating axons (Zhu et al., 1997; Walker et al., 2001). Injury-induced changes in expression of these two genes, although very similar among anamniotes exhibiting successful CNS axon regeneration, are quite different in the amniotes (Mikucki and Oblinger, 1991; Bates and Meyer, 1993; Schaden et al., 1994; Zhao and Szaro, 1995; Rodger et al., 2001; Kaneda et al., 2008). Thus, their expression is tightly coupled with gene regulatory mechanisms controlling the success of CNS axon regeneration.

Temporal changes in expression of both GAP-43 and NF-M during optic nerve regeneration result from a complex interplay between transcriptional and post-transcriptional gene control. RGCs express significant levels of GAP-43 and NF-M nascent primary transcripts, even when axons are not growing, but exhibit increased cytoplasmic mRNA and protein expression only with axon outgrowth (Perrone-Bizzozero et al., 1991; Zhao and Szaro, 1995; Ananthakrishnan et al., 2008). Experiments manipulating the mammalian target of rapamycin pathway, which regulates cap-dependent translation, have demonstrated the importance of increased translation for regeneration (Park et al., 2008, 2010), but RNA-binding proteins that regulate the translation of specific RNAs critical for CNS axon regeneration have not heretofore been described. 
The RNA-binding protein heterogeneous nuclear ribonucleoprotein (hnRNP) K is essential in Xenopus embryos for the initiation of new axons in large part because it is required for the efficient nuclear export and translation of multiple transcripts that collectively organize microfilaments, microtubules, and neuronal intermediate filaments to make the axon (Liu and Szaro, 2011). hnRNP K directly binds both GAP-43 and NF-M RNAs (Irwin et al., 1997; Thyagarajan and Szaro, 2004; Liu and Szaro, 2011), but whether it regulates their expression during optic axon regeneration is unclear, since factors controlling expression of these genes can be distinct as well as shared between development and regeneration (Udvadia et al., 2001; Filbin, 2006; Kusik et al., 2010). Using a novel in vivo method to suppress its expression specifically in RGCs, we show here that hnRNP $\mathrm{K}$ is essential in Xenopus both for optic axon regeneration and for the efficient nuclear export and translation of growth-associated transcripts bound by hnRNP K. Thus, hnRNP $\mathrm{K}$ is an element of a novel posttranscriptional regulatory pathway essential for successful CNS axon regeneration.

\section{Materials and Methods}

Surgery and intraocular injection. For optic nerve crush, juvenile periodic albino Xenopus laevis frogs ( $<6$ months postmetamorphic and of either sex) were anesthetized [0.1\% ethyl 3-aminobenzoate (MS222), SigmaAldrich] and either one (right) or both of their optic nerves were crushed at the orbit as described previously (Zhao and Szaro, 1994). Spinal cord transection was performed as described previously (Gibbs et al., 2011). Triazine-octaguanidinium-modified antisense morpholino oligonucleotides (Vivo-Morpholino, Gene Tools) were intraocularly injected to suppress hnRNP K expression in retinal ganglion cells (RGCs). The VivoMorpholinos (VMOs) used were (1) hnRNP K VMO: 5' GCT GCT ACC TTT CTC CTA CGC CGA C3' targeting nucleotides -54 to -30 (Liu et al., 2008); and (2) 5-nucleotide-mismatch control VMO: 5'GCT CCT AGC TTT CTC GTA CCC CGT C3'. Anesthetized frogs were pressureinjected intraocularly with VMO $(2 \mu \mathrm{l}, 0.5 \mathrm{~mm})$ or saline solution [HEPES-buffered Steinberg's solution (HBS) (Lin and Szaro, 1995)] through a glass micropipette (diameter, $6-8 \mu \mathrm{m}$ ), which was inserted through the cornea, iris, and vitreous until it made direct contact with the retina, slightly injuring the overlying basal lamina. It was then withdrawn slightly above the retinal surface for injection into the vitreous. To maintain hnRNP K knockdown during optic axon regeneration, VMOs were injected $1 \mathrm{~d}$ before nerve crush and then reinjected every $4 \mathrm{~d}$. To trace regenerating axons, $0.5 \mu \mathrm{l}$ of $10 \%$ unconjugated wheat germ agglutinin (WGA; Vector Laboratories) in HBS was intravitreally injected $1 \mathrm{~d}$ before the animal was killed (Zhao and Szaro, 1994).

Immunohistochemistry and in situ hybridization. Anesthetized frogs were perfused with $4 \%$ paraformaldehyde and processed for cryosectioning to yield transverse sections with a thickness of $20 \mu \mathrm{m}$. These sections each contained both sides of the head, as described previously (Gervasi et al., 2003). For immunohistochemistry, primary antibodies ( $\# 1-5$, mouse monoclonal; \#6-9, rabbit antisera; \#10, goat antiserum) were directed against (1) hnRNP K, 1:100 (clone 3C2; Santa Cruz Biotechnology) (Liu et al., 2008; Liu and Szaro, 2011), (2) Xenopus NF-M, $2 \mu \mathrm{g} / \mathrm{ml}$ (RMO270) (Szaro et al., 1989; Wetzel et al., 1989), (3) a Xenopus neuronal- $\beta$-tubulin (N- $\beta$-tubulin) isotype, 1:100 (clone JDR.38B; Sigma-Aldrich) (Moody et al., 1996), (4) GAP-43, 1:500 (clone NM2, Thermo Scientific) (Schrama et al., 1997), (5) proliferating cell nuclear antigen (PCNA), 1:1000 (clone PC10; Sigma-Aldrich) (Gervasi et al., 2000), (6) the conserved carboxy terminal region of tau (tau-3'), 1:2000 (Black et al., 1996; Liu and Szaro, 2011), (7) Xenopus peripherin, 1:2000 (Gervasi et al., 2000), (8) Xenopus hnRNP E2, 1:2000 (Gravina et al., 2002), (9) S6 ribosomal protein, 1:100 (\#2217, Cell Signaling Technology), and (10) WGA, 1:100 (\#AS-2024, Vector Laboratories). 4',6-Diamidino-2-phenylindole (DAPI) and 7 -aminoactinomycin D (7-AAD) $(10 \mu \mathrm{g} / \mathrm{ml}$ in $1 \times \mathrm{PBS}$, Invitrogen $)$ were used to stain cellular nuclei for conventional epifluorescence and confocal laser scanning microscopy (CLSM), respectively. Fluoro-Jade C
$(0.0001 \%$, Millipore $)$ was used to stain degenerating neurons and axons (Chidlow et al., 2009).

For in situ hybridization, digoxigenin-labeled cRNA probes were transcribed [DIG RNA labeling kit (SP6/T7), Roche] from plasmids containing Xenopus NF-M, GAP-43, and peripherin cDNAs (Gervasi et al., 2003; Gibbs et al., 2011). Probe hybridization and visualization, using alkaline phosphatase-conjugated antibodies to digoxigenin, were performed as described previously (Gervasi et al., 2003).

Sections were imaged on either a Zeiss LSM 510 CLSM (20× PlanApoChromat, numerical aperture $0.75 ; 63 \times$ Plan-ApoChromat, numerical aperture 1.4) or a Leitz Laborlux S compound microscope $(25 \times \mathrm{Pl}$ Fluotar, numerical aperture 0.6) equipped with a Nikon DS-Ril camera.

Polysomal profiling. For comparisons of intracellular RNA pools, samples from homogenized eyes were prepared from four conditions ( 5 eyes from 5 animals per condition): (1) animals receiving an optic nerve crush and hnRNP K VMO; (2) animals receiving an optic nerve crush and mismatched control VMO; (3) animals receiving an optic nerve crush without VMO injection; and (4) paired, sham-operated contralateral eyes of Condition 3. Separation of nuclear from cytosolic fractions and subsequent polysomal profiling were performed as described previously (Ananthakrishnan et al., 2008), with the following minor modifications: (1) an SW41 rotor (Beckman Coulter) was used for ultracentrifugation; and (2) the gradient was collected in $0.5 \mathrm{ml}$ fractions using an Auto Densi-Flow density gradient fractionator (Labconco). To quantify nuclear and cytosolic levels of individual mRNAs, total RNA was isolated from the nuclear pellet and from $10 \%$ of the cytosolic fraction (RNeasy Mini Kit, Qiagen). The remainder of the cytosol was used for polysomal profiling. The sequences of primers and TaqMan probes for NF-M, GAP43, and peripherin are provided in Liu and Szaro (2011), and those for Xenopus tau are as follows: forward primer: $5^{\prime}$ GCT TCT TTT ATT TGC CGA TGT TTC3'; reverse primer: 5'AGT ATC AAT GGC AGC AAT GGA A3'; probe: 5'6FAM CCG GCC CCC GTT ATC TTT TTA TTT CAG TA TAMRA3'.

Quantification and statistical analyses. Micrographs quantified for immunofluorescence were imaged by CLSM. For all conditions tested, the images of the experimental and contralateral control retinas to be compared quantitatively originated from the same section, and the imaging parameters (CLSM voxel size, laser intensity, exposure time, and other scanning parameters) were maintained between the two. At the time of collection, images were examined to ensure that pixels were unsaturated. The raw Zeiss LSM images were subsequently exported as TIFF files for analysis by NIS-Elements D 3.10 software (Nikon). To analyze expression levels by immunofluorescence, three nonconsecutive sections taken from the middle third of the eye were analyzed for each animal. All cells of the appropriate layer [either RGC or interneuron (IN)] that could be distinguished as individual cells, as opposed to overlapping cells, were used. To account for variations in cell size, the total fluorescence (arbitrary units) of a region of interest [either the entire cell (see Figs. $2 D, 3$ ) or the nucleus and cytoplasm separately (see Fig. 2C)] was divided by its area to obtain an area-normalized fluorescence intensity $\left(I_{\mathrm{F}}\right)$.

To quantify the nuclear versus cytoplasmic distribution of hnRNP K (see Fig. 2), the limits of the nucleus and cytoplasm were determined by the extent of 7-AAD counterstaining and hnRNP $\mathrm{K}$ immunostaining in each cell, respectively. The area-normalized intensity of hnRNP K staining in the nucleus was then divided by that of the cytoplasm; these data were pooled from three frogs, with each frog contributing approximately the same number of cells. These ratios for RGCs were compared between the unoperated and operated eyes at each time point by $t$ test; numbers of RGCs were 96 and 104 (3 d), and 108 and 113 (11 d) for the unoperated eyes (UEs) and operated eyes (OEs), respectively. Before applying the $t$ test, the quotient of range divided by $\mathrm{SD}(R / S)$ was used to confirm that the distributions satisfied conditions for normality at $\alpha=0.05$ (Pearson and Stephens, 1964; Sachs, 1984).

For analysis of the effects of VMO on the expression of hnRNP K and hnRNP E, the average area-normalized fluorescence intensity per cell of the injected eye was divided by that of the uninjected eye for each section. These ratios were then averaged across the three sections to obtain averages for each animal, which were then averaged among animals in the experiment. A two-tailed $t$ test was then performed to determine whether 

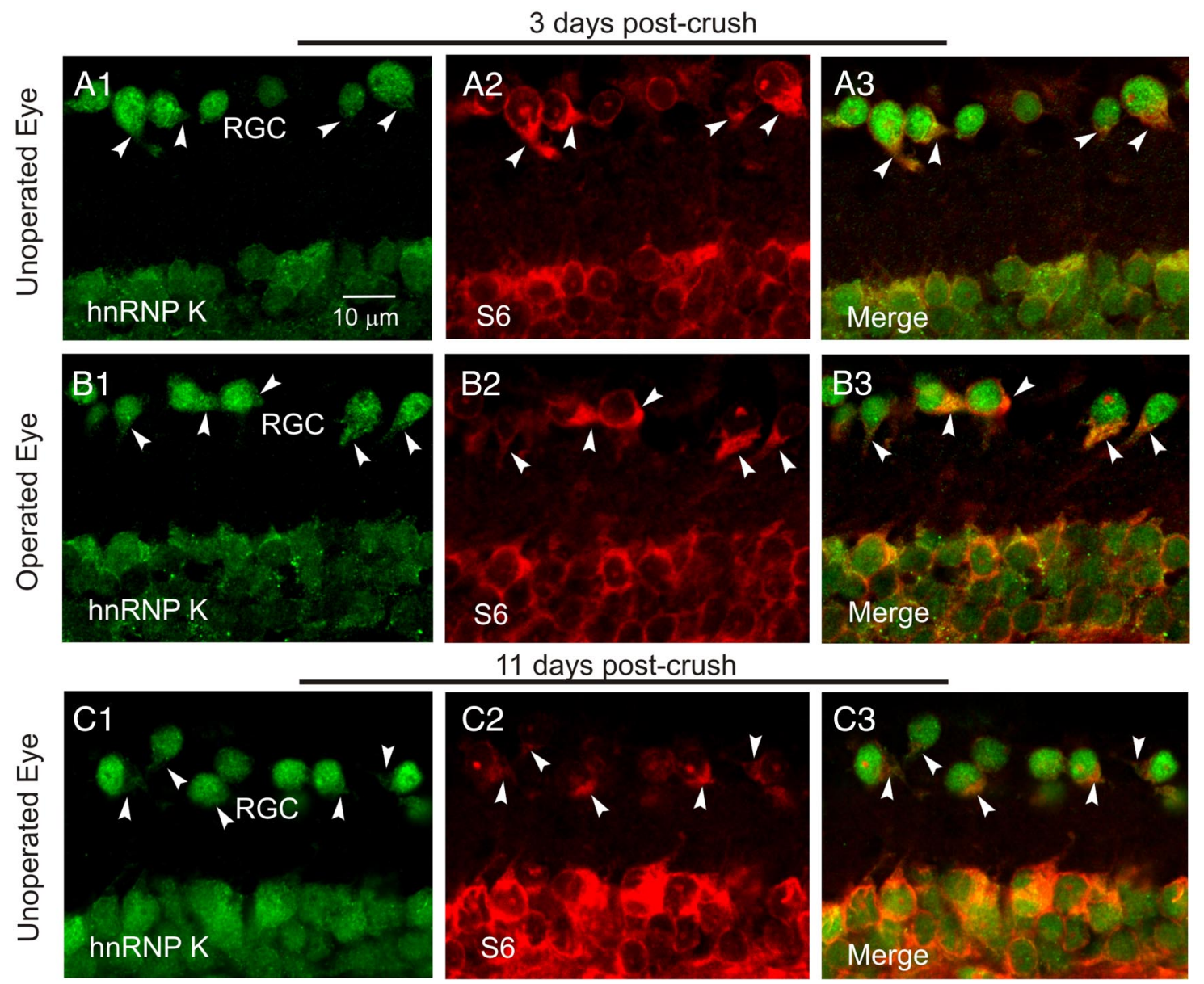

11 days post-crush
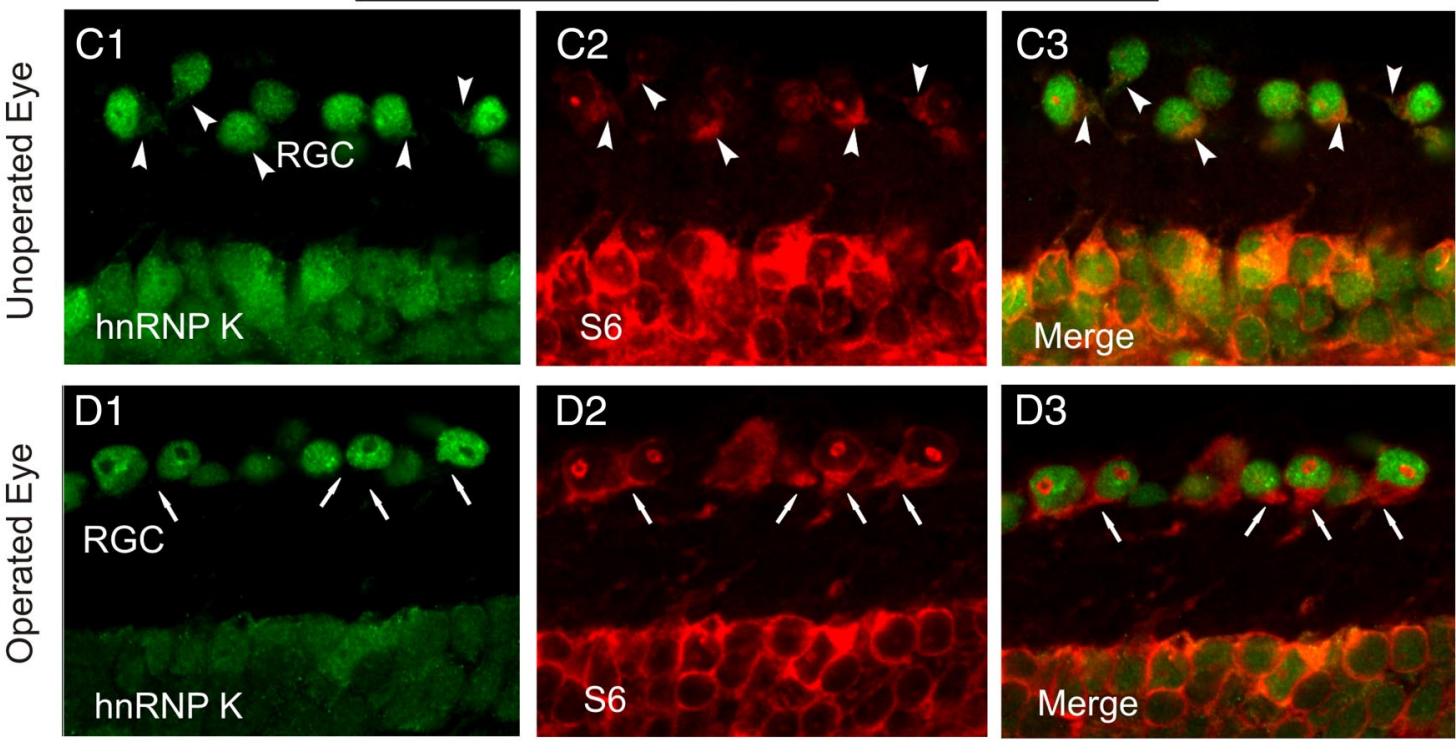

Figure 1. Cytoplasmic versus nuclear localization of hnRNP K shifted in RGCs at the peak period of axon regrowth. A1-D3, Retinal sections were immunostained for hnRNP K (green) and the cytoplasm was counterstained by immunostaining for 56 , a ribosomal protein (red), at early [3 $\mathrm{d}$ after crush (A1-B3)] and peak [11 $\mathrm{d}$ after crush (C1-D3)] phases of axonal regrowth to the optic tectum. CLSM images ( $63 \times$ objective) were taken from the operated and contralateral unoperated eyes within the same section. At $3 \mathrm{~d}$ after crush, immunostaining was comparable between the operated and unoperated eyes, with hnRNP Kimmunoreactivity readily detectable in the cytoplasm as well as in the nucleus of RGCs (A1-B3, arrowheads). At $11 \mathrm{~d}$, hnRNP Kimmunostaining in RGCs of the operated eye was sharply reduced in the cytoplasm and intensified in the nucleus; nuclear staining surrounded a relatively poorly stained nucleolus, positively stained for $\mathbf{5 6}$ (D1-D3, arrows indicate examples of cytoplasmic areas exhibiting significantly reduced hnRNP K staining). In contrast, hnRNP K staining in RGCs of the unoperated eye at $11 \mathrm{~d}$ resembled those of both the operated and unoperated eyes at $3 \mathrm{~d}$ (C1-C3, arrowheads indicate examples of cytoplasmic staining for hnRNP K). Scale bar in $\mathbf{A} 1$ applies to all panels.

these ratios differed significantly from 1.0 (the expected value for no difference between the injected and uninjected eyes), where $n$ was the number of animals. Values were considered statistically significant at $p<$ 0.05 . The total numbers of cells quantified for effects of the hnRNP $\mathrm{K}$ VMO on hnRNP K expression were as follows: 76, 79, 105, and 113 (1 d); 82, 80, 131, and 138 (3 d); 79, 81,102, and 96 (12 d) for uninjected RGC, VMO-injected RGC, uninjected IN, and VMO-injected IN, respectively. The numbers of RGCs quantified for effects of control VMO on hnRNP K expression were 97 and 106 in the injected and uninjected eyes, respectively. The numbers of RGCs quantified for effects of hnRNP K VMO on hnRNP E expression were 88 and 95 for the injected and uninjected eyes, respectively.

For comparisons of the density of 7-AAD-labeled, WGA-labeled, and DAPI-labeled RGCs, an average RGC density per frog was obtained by counting the cells along $100 \mu \mathrm{m}$ of retinal arc from four, nonoverlapping regions of each section and then averaged over three nonconsecutive sections taken from the middle of the eye for each frog ( 12 sample points for each frog). These were then averaged over three separate frogs for each condition. Two-tailed $t$ tests were performed ( $n=$ number of frogs), and results were considered statistically significant at $p<0.05$.

\section{Results}

hnRNP K localization in RGCs shifted from the cytoplasm to the nucleus during optic axon regeneration

In embryonic Xenopus spinal cord, peak periods of axon outgrowth are marked by more intense immunostaining of hnRNP $\mathrm{K}$ in neuronal cell nuclei than in the cytoplasm. As motor neu- 
rons and RGCs mature, cytoplasmic immunostaining intensifies (Liu et al., 2008). To determine whether optic axon regeneration directly induces a change in hnRNP K intracellular localization, fluorescence immunohistochemistry of hn$\mathrm{RNP} \mathrm{K}$ and the ribosomal protein S6 (to label the cytoplasm) was performed at 3 and $11 \mathrm{~d}$ following an orbital optic nerve crush. Consistent with the earlier results in embryos, hnRNP $\mathrm{K}$ immunofluorescence in RGCs of uninjured eyes was clearly present in both the cytoplasm and the nucleus (Fig. 1A1-3,C1-3). With injury, the intracellular distribution was similar between the operated and contralateral unoperated eye at $3 \mathrm{~d}$ (Fig. 1 A1$B 3)$, when regenerating axons had not yet crossed the lesion (Zhao and Szaro, 1994). However, at $11 \mathrm{~d}$ (Fig. 1C1-D3), when regrowing axons are traversing the optic tract (Zhao and Szaro, 1994), hnRNP K immunofluorescence in RGCs of the operated eye shifted decidedly into the nucleus to resemble more closely the situation in newly developing neurons during peak axonal outgrowth (Liu et al., 2008), whereas RGCs of the unoperated eye more closely resembled those at $3 \mathrm{~d}$. No such shift occurred in other retinal cell types. To confirm this shift quantitatively, ratios of the intensity of immunofluorescence in the nucleus to that of the cytoplasm were determined for RGCs (Fig. 2). This ratio increased $>5$-fold in the operated eye at $11 \mathrm{~d}$ over that seen in either the unoperated eye at $11 \mathrm{~d}$ or in both eyes at $3 \mathrm{~d}$ (Fig. 2C). Moreover, this shift occurred in the absence of any significant changes in overall levels of expression of hnRNP K within RGCs (Fig. 2D), further confirming that the changes observed represented shifts in intracellular localization from the cytoplasm to the nucleus (Fig. 1D1-D3).

\section{hnRNP K expression was specifically suppressed in RGCs by VMO injection}

To study hnRNP K's function during optic axon regeneration, we used intravitreal injection of VMO. The sequence of the hnRNP K VMO was identical to the sequence of a conventional antisense morpholino used previously to suppress hnRNP K expression in Xenopus embryos (Liu et al., 2008; Liu and Szaro, 2011). Effects on hnRNP K expression in the VMO-injected uninjured eye were quantified by comparing hnRNP K immunofluorescence with that in the contralateral uninjected eye (area normalized $I_{\mathrm{F}}$ per cell in the injected eye divided by that of the uninjected eye). At $1 \mathrm{~d}$ after injection, hnRNP K expression in RGCs of the injected eye fell to $19 \pm 1 \%$ (SEM) that of the contralateral uninjected eye in the same section (Fig. $3 A 1, A 2, E$ ). By $3 \mathrm{~d}$, expression in RGCs of the $\mathrm{VMO}$-injected eye had risen slightly to $27 \pm 1 \%$ that of the contralateral uninjected eye (Fig. $3 B 1, B 2, E$ ), but by repeating injections every $4 \mathrm{~d}$, knockdown could be maintained within this range for at least $12 \mathrm{~d}(29 \pm 1 \%$, Fig. 3C1,C2,E). hnRNP K knock-
$7-A A D$
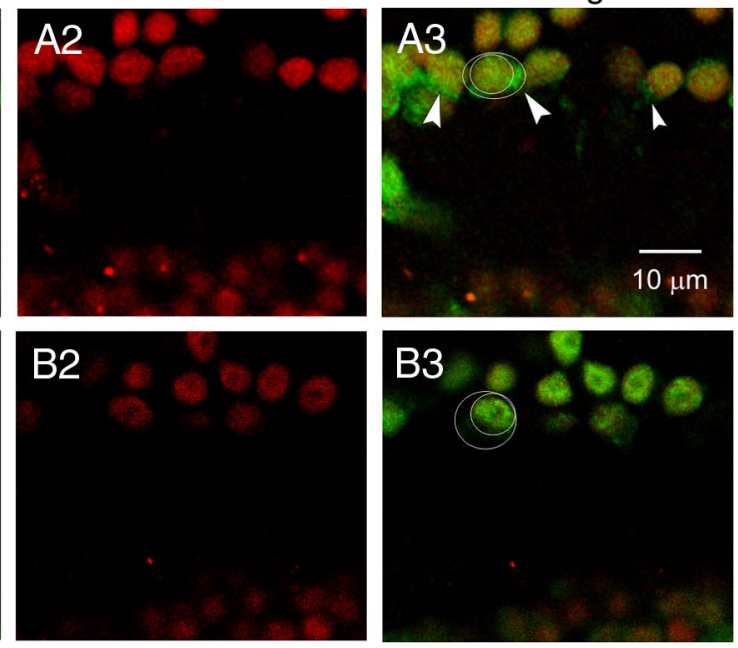

$D$ Relative fluorescence intensity $\left(I_{F}\right)$ of whole cell hnRNP K in RGCs of OE
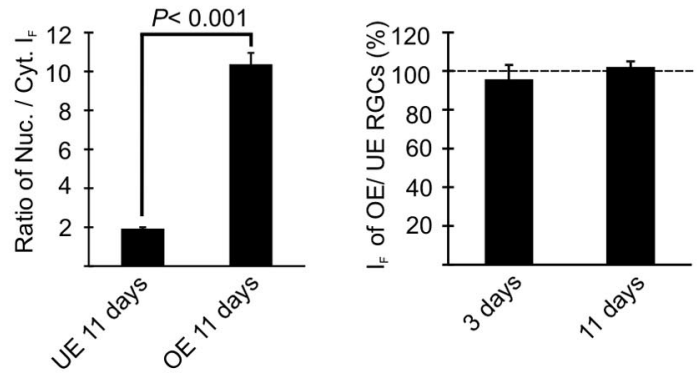

Figure 2. Quantification of nuclear versus cytoplasmic localization of hnRNP K during regeneration. A1-B3, Representative sections of retina (11 $d$ after crush) immunostained for hnRNP K (green) and nuclear counterstained with 7-AAD (red) from the operated (B1-B3) and contralateral unoperated $(\boldsymbol{A} 1-\boldsymbol{A} \mathbf{3})$ eyes within the same section. Circles $(\boldsymbol{A} \mathbf{3}, \boldsymbol{B} \mathbf{3})$ indicate examples of the intensities were not significantly different between RGCs of the $0 \mathrm{E}$ versus those of the contralateral UE at either time point [OE/UE ( \pm SEM): $3 \mathrm{~d}, 96 \pm 7 \%, p=0.5 ; 11 \mathrm{~d}, 103 \pm 3 \%, p=0.7 ; t$ test, $n=3$ animals $]$.

down was specific to and uniformly distributed across the entire RGC layer [Fig. 3A1-C2,F; for interneurons (injected eye/uninjected eye): $1 \mathrm{~d}, 96 \pm 4 \% ; 3 \mathrm{~d}, 94 \pm 5 \% ; 12 \mathrm{~d}, 91 \pm 7 \%]$. This restriction of hnRNP K knockdown to RGCs ensured that effects on optic axon regeneration were intrinsic to RGCs and not caused by interactions between RGCs and other retinal cell types.

Continued injection of hnRNP K VMO over 12 days induced neither reductions in RGC number (determined by counting 7-AAD-counterstained nuclei; Fig. 3D1,D2,H), nor axonal degeneration (determined by tau immunostaining of optic nerve, Fig. 3J1,J2), nor activation of peripherin expression in RGCs, which is normally indicative of optic axon injury (Fig. $3 \mathrm{~K} 1, \mathrm{~K} 2$; in uninjured retina, peripherin immunostaining is restricted to Mueller glia). Thus, exposure to VMO was not overtly cytotoxic. Specificity of the conventional hnRNP K morpholino of the same sequence was previously demonstrated in Xenopus embryos both by injection of a second, nonoverlapping morpholino and by a rescue experiment in which the morpholino was coinjected with hnRNP K RNA (Liu et al., 2008); we confirmed that knockdown by VMO was also specific in retina by demonstrating that a control VMO whose sequence differed from that of the hnRNP K 

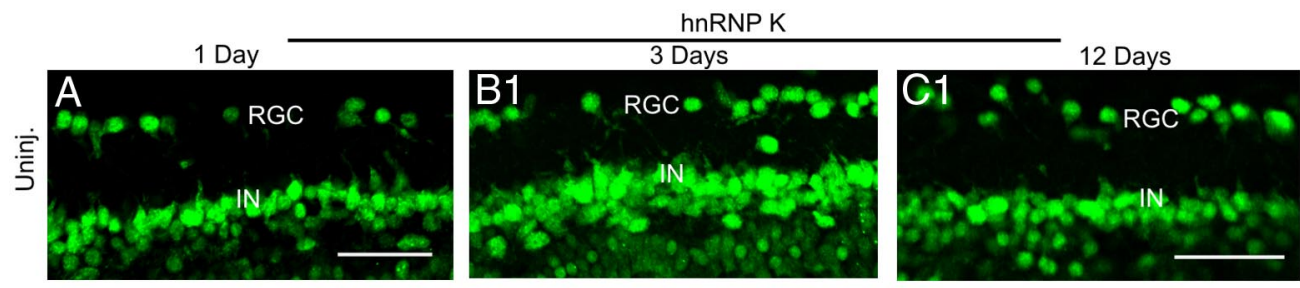

7-AAD
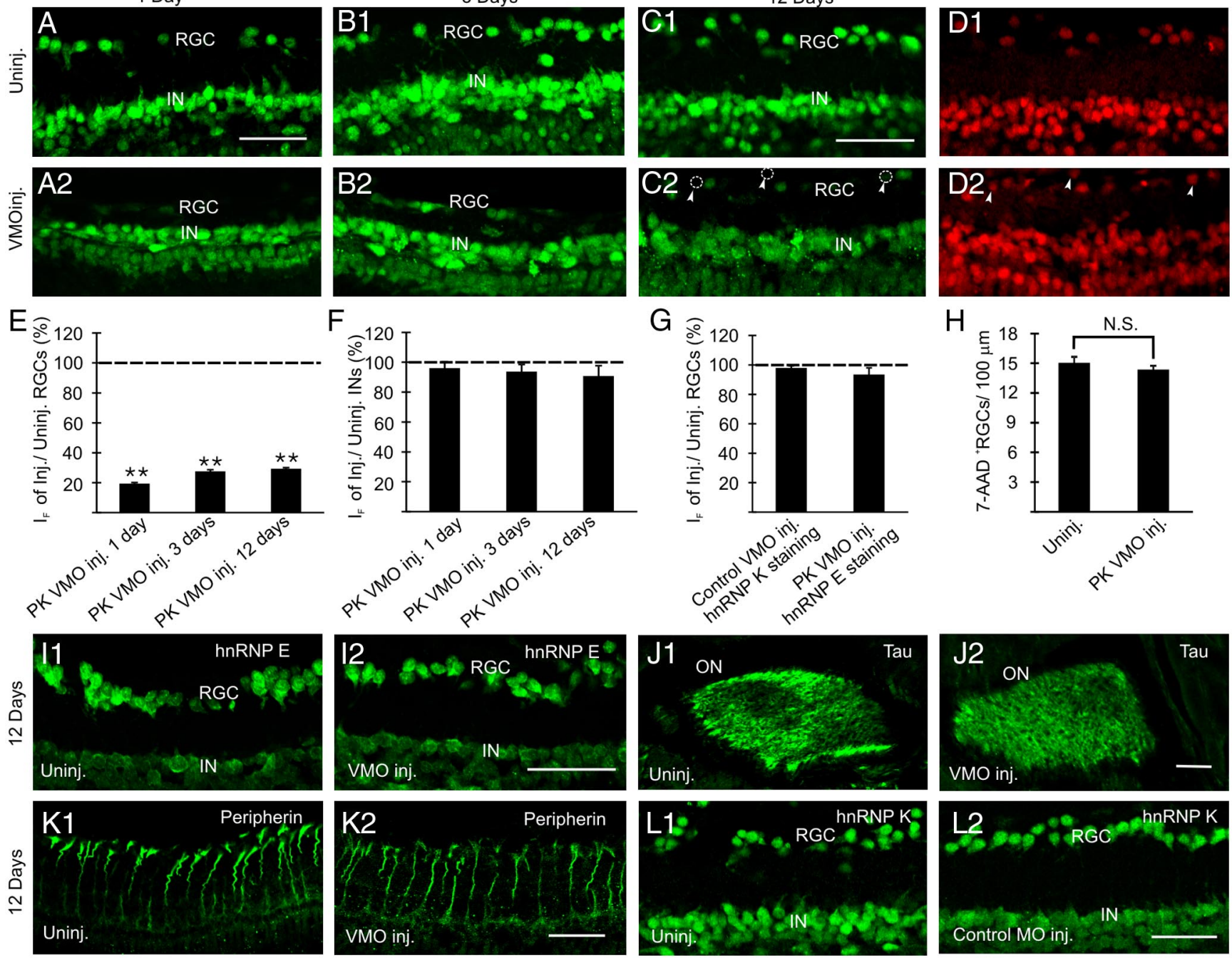

Figure 3. Antisense VMO suppressed hnRNP K expression in RGCs. Following repeated, unilateral, intravitreal injection of VMO, unoperated frogs were killed at the indicated time points, processed for immunofluorescence, and viewed by CLSM. A1-C2, Severe depletion of hnRNP K expression from RGCs, but not INs in eyes injected with hnRNP K VMO (lower micrograph, VM0 inj.) compared to the contralateral uninjected eye (upper micrograph, Uninj.). D1, D2, Nuclear counterstaining (7-AAD) of retinal sections depicted in C1 and C2. Arrowheads indicate examples of RGCS in which hnRNP K protein expression was undetectable by immunostaining ( $(2$, Arrowheads with dotted lines circumscribing the position of cell). $\boldsymbol{E}$, $\boldsymbol{F}$, Quantification of the ratio of hnRNP $K$ immunofluorescence intensity per cell between the hnRNP KVMO-injected (PK VMO inj.) and uninjected eyes (mean \pm SEM\%) confirmed that suppression of hnRNP K expression was significant at all time points for $\mathrm{RGCS}\left(\boldsymbol{E}, p<0.001, t\right.$ test, $n=4$ animals) but not for interneurons $\left(\boldsymbol{F}, p=0.3,0.2\right.$, and 0.2, for 1,3 , and $12 \mathrm{~d}$, respectively, $t$ test). $\boldsymbol{H}$, Density (average number of $7-\mathrm{AAD}^{+} \mathrm{RGCS} / 100$ $\mu \mathrm{m}$ of retinal arc per animal \pm SD) of 7-AAD-labeled nuclei in the RGC layer was not significantly different between hnRNP KVM0-injected (VMO inj.) and uninjected (Uninj.) eyes at $12 \mathrm{~d}(p=0.29$, $t$ test). I1-K2, Immunofluorescence indicated that hnRNP K VMO (12 d) affected neither the expression of hnRNP E2 (II, $\mathbf{2})$ and peripherin $(\boldsymbol{K 1}, \boldsymbol{K} \mathbf{2})$ in retina nor of tau $(\boldsymbol{J 1}, \boldsymbol{J} \mathbf{2})$ in optic nerve. $\mathbf{L} \mathbf{1}$, $\mathbf{L 2}$, Mismatched control VMO (12 d) had no effect on hnRNP K immunofluorescence in RGCs. G, Quantification of data illustrated in $\mathbf{I 1}, \mathbf{I 2}, \mathbf{L} \mathbf{1}$, and $\mathbf{L} \mathbf{2}$ (Inj. eye/Uninj. eye; mean \pm SEM; $p=0.2$ for $\mathbf{I 1}$ and $\mathbf{2} ; \mathrm{p}=0.25$ for $\mathbf{L} \mathbf{1}$ and $\mathbf{L 2} ; \boldsymbol{t}$ test, $n=3$ animals). Scale bars, $50 \mu \mathrm{m}$. Scales bar in $\mathbf{A} \mathbf{1}$ also applies to $\mathbf{A 2}-\mathbf{B 2}$; scale bar in $\mathbf{C 1}$, also applies to $\mathbf{C 2}-\mathbf{D 2}$; scale bar in $\mathbf{2}$, also applies to $\mathbf{1 1}$; scale bar in $\mathbf{J} \mathbf{2}$ also applies to $\mathbf{J 1}$; scale bar in $\mathbf{K} \mathbf{2}$ also applies to $\boldsymbol{K 1}$; scale bar in $\mathbf{L} \mathbf{2}$ also applies to $\mathbf{L 1}$.

VMO by five nucleotides ( $5 \mathrm{nt}$ mismatch control) had no significant effect on hnRNP K expression at $12 \mathrm{~d}$ (Fig. 3L1,L2,G; injected eye/uninjected eye RGC: $98 \pm 2 \%$ ). Further, it was unlikely that genes other than hnRNP K were targeted, since there was no significant effect on expression of Xenopus hnRNP E2 (Fig. $3 I 1, I 2, G$; injected eye/uninjected eye RGC: $94 \pm 5 \%$ ), the RNAbinding protein in Xenopus that is most closely related to Xenopus hnRNP K (Ostareck-Lederer et al., 1998; Gravina et al., 2002; Busch and Hertel, 2012).

Suppression of hnRNP K expression in RGCs inhibited optic axon regeneration

We next examined effects of hnRNP K knockdown on optic axon regeneration. To maintain suppression, we injected hnRNP K
VMO into one eye $1 \mathrm{~d}$ before performing optic nerve crush on both eyes and repeated the injection every $4 \mathrm{~d}$. At $12 \mathrm{~d}$ after crush, we assayed the progress of regeneration on both sides by immunostaining for an $\mathrm{N}-\beta$-tubulin isotype, which labels axons and whose RNA is not a target of hnRNP K. The time course of optic nerve regeneration after an orbital optic nerve crush, as well as the distribution of regenerating and degenerating axons within the nerve, is well characterized in Xenopus frogs (Jacobson, 1961; Fawcett and Gaze, 1981; Szaro et al., 1985; Goodbrand and Gaze, 1991; Wilson et al., 1992; Zhao and Szaro, 1994). On the uninjected side, the distribution of regenerating fibers was typical for $12 \mathrm{~d}$ of optic nerve regeneration, when the front of regeneration lies within the optic tract; regenerating axons penetrated the lesion site and, within the optic nerve itself, they circumscribed the 


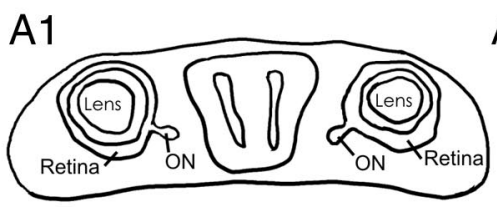

Uninjected
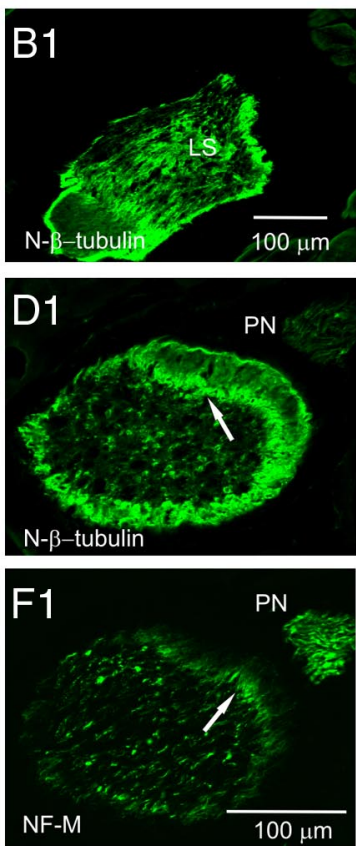

Uninjected

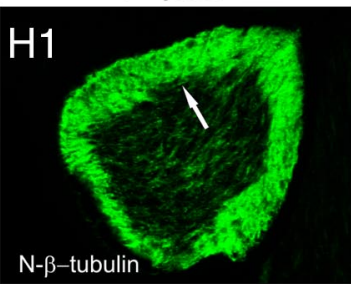

hnRNP K VMO injected
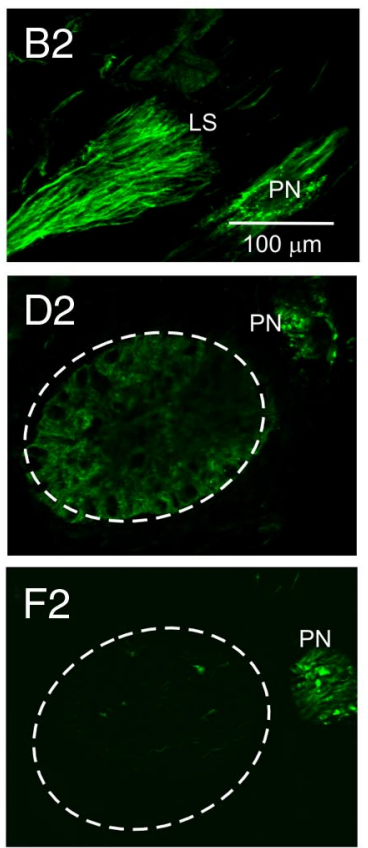

Control VMO injected

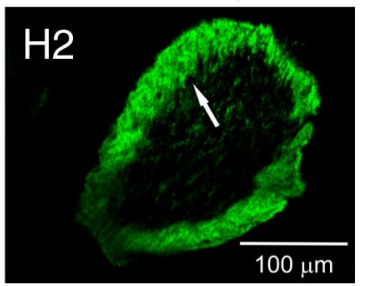

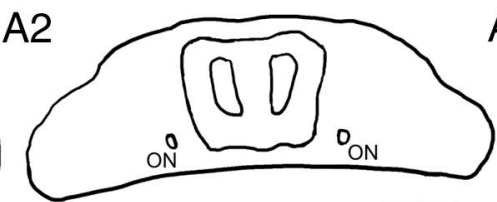

$\overline{1 \mathrm{~mm}}$

Uninjected
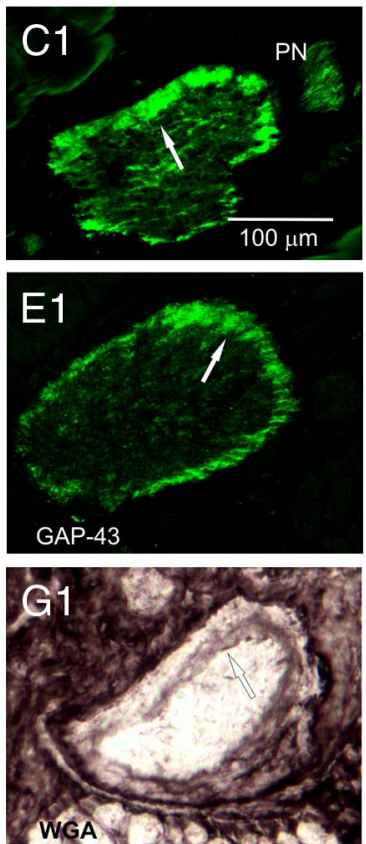

Uninjected

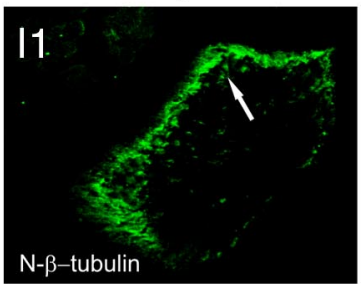

hnRNP K VMO injected
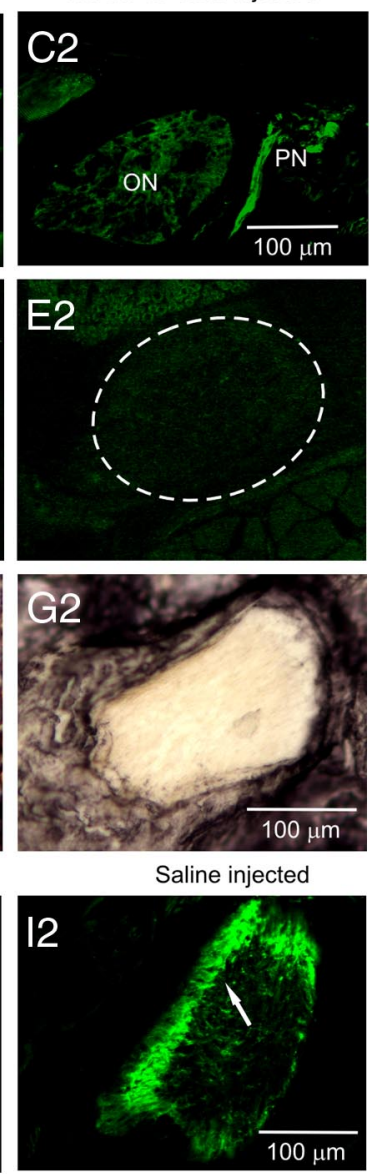

Figure 4. hnRNP K knockdown inhibited optic axon regeneration. B1- I2, Animals were subjected to orbital optic nerve crush on both sides but injected with hnRNP K VMO (B2, C2, D2, E2, F2, G2), mismatched control VMO (H2), or saline (I2) on only one side. Animals were processed for immunostaining at $12 \mathrm{~d}$ after crush. $\boldsymbol{A} 1-\boldsymbol{A 3}$, Tracings of representative transverse sections of the optic nerve at progressively more caudal levels from where the optic nerve exits the eye $(\boldsymbol{A} \mathbf{1})$ to the optic chiasm $(\boldsymbol{A} \mathbf{3})$. Approximately 36 sections are between $\boldsymbol{A} \mathbf{1}$ and $\boldsymbol{A 2}$. Approximately 52 sections are between $\mathbf{A} \mathbf{2}$ and $\mathbf{A 3}$. B1-I2, Images of the optic nerve were taken from either near the lesion site (B1-C2, at the position illustrated in $\boldsymbol{A}$ ) or near the position illustrated in $\boldsymbol{A 2}$ (D1-I2). All images of the injected and uninjected sides were taken from the same sections. $\boldsymbol{B} 1, \mathbf{C}$, 0 n the uninjected side, $\mathrm{N}$ - $\beta$-tubulin-positive regenerating optic axons penetrated the lesion site (B1, $L S)$ to occupy the periphery of the optic nerve in the immediately adjacent section ( $\mathbf{C}$, arrow). $\mathbf{B 2}$, $\mathbf{C}$, On the hnRNP K VM0-injected side, axons stopped at the lesion site (B2, LS) and could not be detected in the section immediately beyond [ $\mathbf{C}, \mathbf{O N}$ (optic nerve); staining of the adjacent peripheral nerve (PN) indicates that the immunostaining procedure was successful]. D1-G2, Immunostaining of hnRNP K VM0-injected animals for axonal markers [N- $\beta$-tubulin $(\mathbf{D 1}, \mathbf{D 2})$, GAP-43 (E1, E2), NF-M (F1, F2) by immunofluorescence] or the intravitreally injected axonal tracer WGA (G1, G2; by immunoperoxidase). On the uninjected side, labeled regenerating axons circumscribed the core of degenerating axons (arrows) but were undetectable on the injected side (dotted ellipse indicates outer circumference of the optic nerve). $\boldsymbol{H 1}-\mathbf{I 2}, \mathrm{N}-\beta$-tubulin staining of regenerating optic axons of control VM0-injected $(\boldsymbol{H 1}, \boldsymbol{H} \mathbf{2})$ or saline-injected $(\boldsymbol{I 1}, \boldsymbol{I 2})$ animals at $12 \mathrm{~d}$ after crush indicated that these treatments had no effect on regeneration. Scale bar in $\boldsymbol{F} \mathbf{1}$ also applies to $\mathbf{D 1}-\mathbf{E 2}$ and to $\mathbf{F 2}$; scale bar in $\mathbf{G 2}$ also applies to $\mathbf{G 1}$; scale bar in $\boldsymbol{H 2}$ also applies to $\boldsymbol{H 1}$; scale bar in $\mathbf{2}$ also applies to I1.

core of the optic nerve, which contains the degenerating axons (Fig. $4 B 1, C 1, D 1$ ). In stark contrast, on the hnRNP K VMOinjected side, $\mathrm{N}-\beta$-tubulin-stained optic axons stopped abruptly at the lesion site, failing to penetrate it and leaving the optic nerve beyond entirely devoid of regenerating axons (Fig. 4B2,C2,D2). Immunostaining for NF-M and GAP-43, two additional axonal markers whose RNAs are targets of hnRNP K, exhibited a similar pattern (Fig. 4E1-F2). To confirm that the absence of immunostaining for axonal markers from the optic nerve of VMOinjected eyes was an indication that axons had failed to regenerate, we injected WGA, an anterograde axonal tracer,
$1 \mathrm{~d}$ before killing the animal, into both the VMO-injected and uninjected eyes, and detected it in optic nerve sections by immunoperoxidase (Zhao and Szaro, 1994). WGA-labeled regenerating axons were seen in the optic nerve of only the uninjected eye (Fig. 4G1,G2), even though uptake of WGA was unaffected by hnRNP K knockdown (see below, hnRNP K knockdown did not increase neurodegeneration during regeneration). Also, the effect on regeneration was specific to the hnRNP K VMO because neither control VMO injections nor saline injections had any effect on $\mathrm{N}$ - $\beta$-tubulin staining of regenerating optic axons (Fig. 4H1-I2). 
N- $\beta$-tubulin

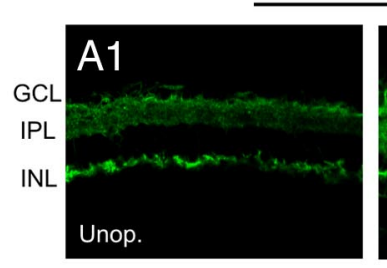

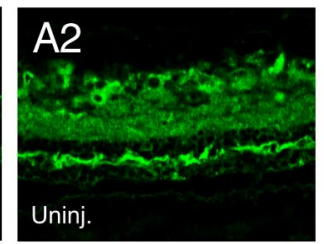
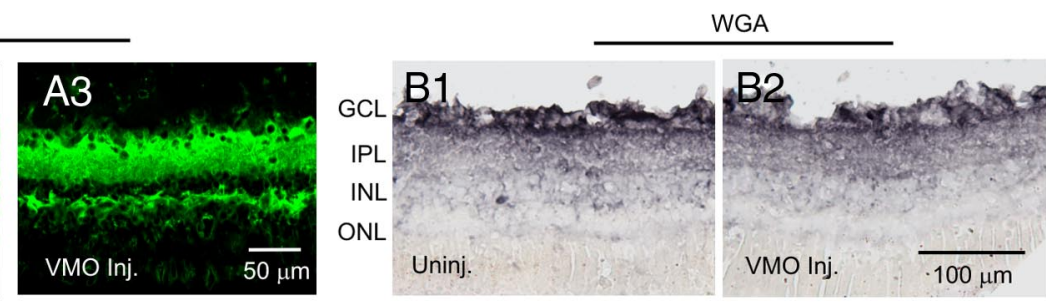

Fluoro-Jade $\mathrm{C}$
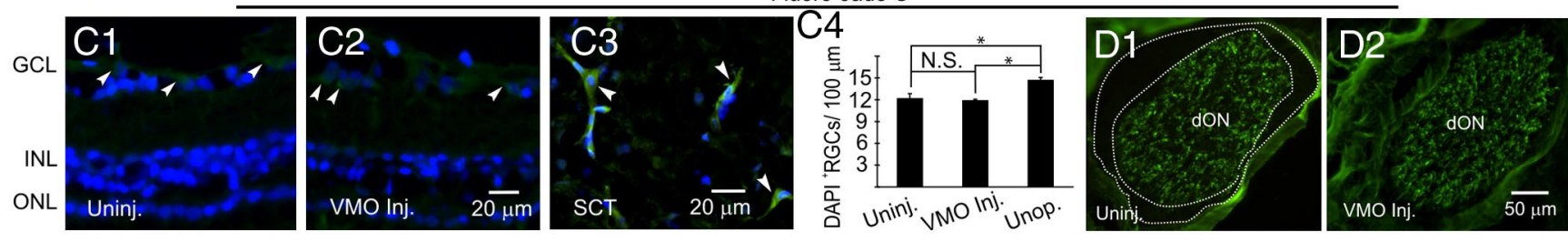

Figure 5. hnRNPK knockdown by itself did not induce neurodegeneration during optic axon regeneration. Frogs receiving bilateral, orbital optic nerve crushes and unilateral injections of hnRNP K VM0 were killed at $12 \mathrm{~d}$ after crush. $\boldsymbol{A} \mathbf{1}, \boldsymbol{A 2}, \boldsymbol{A 3}$, Immunostaining for $\mathrm{N}$ - $\beta$-tubulin in the ganglion cell $(\mathrm{GCL})$ and inner plexiform (IPL) layers was more robust at $12 \mathrm{~d}$ after crush (A2, $\boldsymbol{A} \mathbf{3})$ than in surgically naive, unoperated eye (A1) and in the hnRNP K VM0-injected eye (A3) than in the contralateral uninjected eye (A2). B1, B2, Uptake of WGA in RGCs was unchanged in the hnRNP K VM0-injected eye (B2), compared with the contralateral uninjected eye (B1). The density of WGA-labeled RGCs (RGCS/100 $\mu \mathrm{m}$ of retinal arc \pm SD) was not significantly different between the uninjected and injected eyes (11.6 \pm 0.3 vs $11.4 \pm 0.5$, respectively; $p=0.28, t$ test) (B2 became cropped in the lower right hand corner when the image was rotated). $\mathbf{C}$ - $\mathbf{C}$, Fluoro-Jade $\mathbf{C}$ staining was similar between the retina of hnRNP K VM0-injected (C2) and uninjected (C1) eyes. In both eyes, Fluoro-Jade C staining of retina was much less intense than in cells adjacent to a spinal cord transection (C3). C4, Density of DAPI-labeled nuclei in the retinal ganglion cell layer (DAPI ${ }^{+} \mathrm{RGCS} / 100 \mu \mathrm{m}$ of retinal arc \pm SD) was not significantly different between hnRNP K VM0-injected (VM0 inj.) and uninjected (Uninj.) eyes (N.S., $p=0.12, t$ test); although both exhibited a significant decrease (19\% loss; ${ }^{*} p<0.01$, $t$ test on counts derived from 3 frogs) compared with the uninjured eye. D1, D2, Fluoro-Jade ( staining of degenerating optic axons (d0N) was similar between VM0-injected (D2) and uninjected (D1) eyes. D1, Dotted outline indicates the positions of regenerating axons, unstained by Fluoro-Jade C, surrounding the degenerating core. No such region is visible on the VM0-injected side. INL, inner nuclear layer; ONL, outer nuclear layer. Scale bar in $\mathbf{A} \mathbf{3}$ applies to $\mathbf{A 1}$ and $\mathbf{A 2}$; scale bar in $\mathbf{B 2}$ applies to $\mathbf{B 1}$; scale bar in $\mathbf{C 2}$ applies to $\mathbf{C 1}$; scale bar in D2 applies to D1.

\section{hnRNP K knockdown did not increase neurodegeneration during regeneration}

We used several measures to determine whether hnRNP $\mathrm{K}$ knockdown adversely affected the vitality of RGCs during optic axon regeneration. First, we immunostained retina at $12 \mathrm{~d}$ after crush for two proteins that are normally upregulated during optic axon regeneration and whose RNAs are not targets of hnRNP K, namely $\mathrm{N}$ - $\beta$-tubulin and peripherin (Heacock and Agranoff, 1976; Gervasi et al., 2003). Expression of these two proteins increased during optic nerve regeneration in the hnRNP K VMOinjected eye (Figs. 5A1-A3 and 6E1-E3); interestingly, the increase of $\mathrm{N}$ - $\beta$-tubulin was greater in the VMO-injected eye than in the uninjected eye (Fig. 5A2,A3), possibly due to the accumulation of protein in RGC perikarya in the absence of a regenerating axon into which it could be transported. Second, uptake of intravitreally injected WGA into RGCs was comparable in both eyes, indicating that RGCs in the VMO-injected eye remained sufficiently healthy to take up the exogenous lectin (Fig. $5 B 1, B 2)$. Third, staining of retina with Fluoro-Jade C, a marker for neuronal degeneration (Schmued et al., 2005; Chidlow et al., 2009), was comparable between the uninjected and injected eye, although markedly reduced in both eyes compared with that seen in spinal cord after transection (Fig. 5C1-C3). Despite very low levels of Fluoro-Jade $\mathrm{C}$ staining in retina, the degenerating axons in the core of the optic nerve on both sides of the animal were intensely stained (Fig. 5D1,D2). Fourth, cell counts of DAPIlabeled nuclei in the RGC layer were comparable between injected and uninjected eyes. The densities of RGCs on both sides were reduced equally $(\sim 20 \%)$ compared with unoperated eye (Fig. 5C4). These lack of differences between VMO-injected and uninjected eyes during regeneration was not because the VMO had induced abnormal cell proliferation, since immunostaining for PCNA, a marker for proliferating cells (Waseem and Lane, 1990; Leibovici et al., 1992; Gervasi et al., 2000), showed no labeling in either retina (data not shown). These data indicated that the survival rate of RGCs was not significantly affected by hnRNP $\mathrm{K}$ knockdown during optic axon regeneration.

hnRNP K knockdown by VMO inhibited protein, but not mRNA, expression of select cytoskeletal-associated genes during optic axon regeneration

In newly developing neurons, hnRNP K is required for the translation into protein of multiple, functionally interrelated mRNAs that collectively are needed for axon initiation and subsequent outgrowth (Liu et al., 2008; Liu and Szaro, 2011). We selected two previously validated hnRNP K targets, NF-M and GAP-43 (Irwin et al., 1997; Thyagarajan and Szaro, 2004; Liu et al., 2008; Liu and Szaro, 2011), to test whether hnRNP K is similarly required for their expression during optic axon regeneration. $N F-M$ and $G A P-43$ expression normally increase dramatically during optic axon regeneration, and this increase involves significant control at the post-transcriptional level (Benowitz et al., 1981; Skene and Willard, 1981; Perrone-Bizzozero et al., 1991; Gervasi et al., 2003; Ananthakrishnan et al., 2008). To control for the specificity of effects of hnRNP K knockdown, we also examined peripherin, a neuronal intermediate filament gene whose expression increases dramatically in RGCs during optic nerve regeneration, but whose mRNA is not a target of hnRNP K (Gervasi et al., 2003). Consistent with these earlier studies, both mRNA and protein expression of NF-M and GAP-43 increased dramatically at $12 \mathrm{~d}$ after crush in RGCs of operated uninjected eyes compared with the contralateral, unoperated control eye (Fig. 6A1,A2,B1,B2,C1,C2,D1,D2). In hnRNP K $\mathrm{VMO}$-injected eyes, however, mRNA expression increased relative to the uncrushed control eye, but protein expression was severely diminished (Fig. 6A3,B3,C3,D3). These observations indicated that RGCs of hnRNP K VMO-injected eyes responded to the injury by increasing mRNA expression of several axonalgrowth-associated mRNAs, but that proper post-transcriptional regulation of specifically the hnRNP K-targeted RNAs leading to 
increased protein expression required hnRNP K. In contrast, hnRNP K knockdown had no effect on the injury-induced, increased expression of peripherin protein in RGCs (Fig. 6E1-F3), which in uninjured eye is normally seen only in Mueller glia, indicating that the defect was not a generalized one.

hnRNP K knockdown induced defects in nuclear export and translation of targeted cytoskeletal-associated transcripts during optic axon regeneration

During development, hnRNP K knockdown significantly diminishes the efficiency of nuclear export of several cytoskeletalassociated RNAs, including NF-M and tau (Liu and Szaro, 2011). To examine whether hnRNP K knockdown led to similar deficiencies during regeneration, we performed the same qRT-PCR assay to determine the relative amounts of $N F-M$, GAP-43, tau, and peripherin RNA present in nuclear versus cytosolic fractions of eyes at $12 \mathrm{~d}$ after crush (Fig. $7 A$ ). The $\Delta C_{\mathrm{t}}$ (difference in the number of cycles to reach threshold) was significantly reduced for $N F-M$ [23-fold $\left.\left(2_{\mathrm{t}}^{\Delta \mathrm{C}}\right), p=0.004\right]$, GAP-43 (6-fold, $p=0.03$ ), and tau (2fold, $p=0.05)$ RNAs, but not for peripherin RNA, in hnRNP K VMO-injected eyes compared with control VMO-injected eyes during regeneration. Variations in the magnitude of these defects among the three affected RNAs were likely due to differences in the degree to which their expressions were restricted to RGCs relative to other retinal cell layers, since hnRNP K expression was affected only in the ganglion cell layer. Whereas NF-M RNA is exclusively expressed in RGCs (Fig. 6B1-B3), GAP-43 RNA is also seen in scattered cells within the inner nuclear layer (Fig. 6D1-D3), and tau is expressed in all retinal cell layers (data not shown).

Despite a statistically significant decrease in the efficiency of nuclear export, substantial amounts of targeted mRNAs were nevertheless exported to the cytoplasm. To test whether the failure of protein to be expressed involved direct effects of hnRNP K knockdown on translation of these mRNAs, we performed polysomal profiling on cytoplasmic extracts (Fig. $7 B, C$ ). In this differential ultracentrifugation assay, the most actively translated mRNAs fractionate with polysomes, whereas translationally silent mRNAs fractionate with monosomes and lighter fractions. Consistent with earlier reports for NF-M and peripherin mRNAs (Ananthakrishnan et al., 2008; Ananthakrishnan and Szaro, 2009), they, as well as GAP-43 and tau mRNAs, were more translationally active in uninjected operated eye than in shamoperated eye at $12 \mathrm{~d}$ after crush (63 vs $23 \%, 54$ vs $11 \%, 49$ vs $20 \%$, and 27 vs $10 \%$ for NF-M, GAP-43, tau, and peripherin, respectively), indicating that all these mRNAs were under strong translational control during regeneration. This injury-induced shift

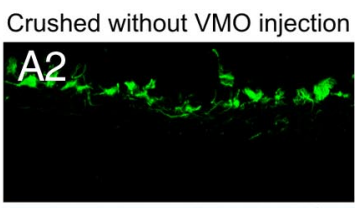

Crushed with VMO injection
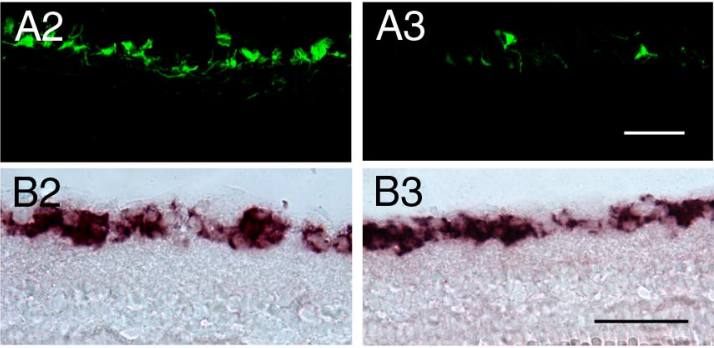

B3
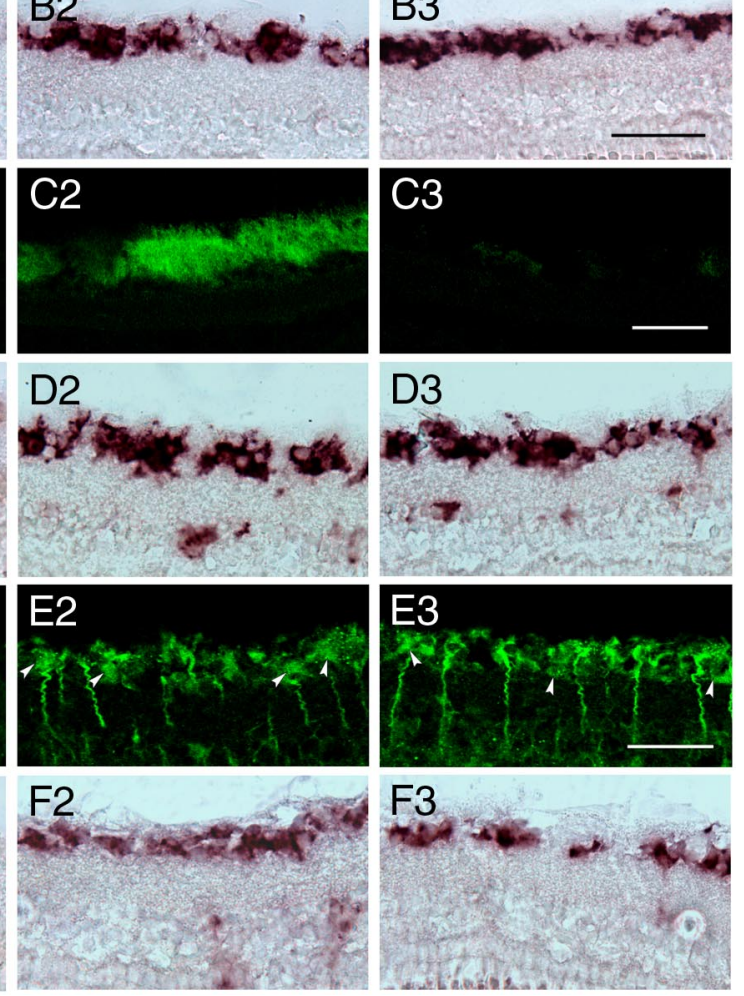

Figure 6. hnRNP K knockdown inhibited protein, but not mRNA, expression of select cytoskeletal-associated targets activated during optic nerve regeneration. A1-F3, Sections containing hnRNP K VM0-injected and contralateral uninjected eyes from animals receiving bilateral optic nerve crush $12 \mathrm{~d}$ earlier, and sections of uninjected eyes receiving no crush were processed for 作 ization (B1-B3, D1-D3, F1-F3; imaged in brightfield) to compare protein with mRNA expression for NF-M (A1-B3), GAP-43 D3), and peripherin (E1-F3). mRNA expression of all three genes increased dramatically in the $\mathrm{GCL}$ with optic nerve crush,

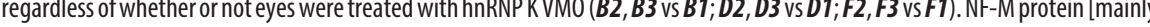
visible in RGC axons, in the nerve fiber layer (NFL)] and GAP-43 protein (mainly present in RGC dendrites, located in the IPL), Scale bars, $50 \mu \mathrm{m}$. Scale bar in $\mathbf{A 3}$ applies to A1, A2; scale bar in C3 applies to C1, C2; scale bar in E3 applies to E1, E2; scale bar in B3 applies to $B$ 1, B2, D1-D3, F1-F3.

from poorly translated to efficiently translated fractions was significantly compromised for all three hnRNP K-targeted mRNAs with the hnRNP K VMO but not with the control VMO (NF-M, 12 vs $58 \%$; $G A P-43,8$ vs $51 \%$; tau, 18 vs $45 \%$, respectively, $p<$ $0.01, t$ test). The increased presence of the nontargeted mRNA (peripherin) in polysomes during regeneration was unaffected by hnRNP K knockdown $(23,26$, and $27 \%$ in polysomal fractions for hnRNP K VMO-injected, control VMO-injected, and uninjected operated eye, respectively; $p>0.1, t$ test), indicating that the defect in translation was specific to the hnRNP K-targeted RNAs.

\section{Discussion}

Our data demonstrate that hnRNP K is an essential component of a novel pathway regulating the intrinsic response to injury that leads to successful CNS axon regeneration. At the molecular level, hnRNP K was specifically required to promote polysome loading, as well as efficient nuclear export, of mRNAs of several axonal 


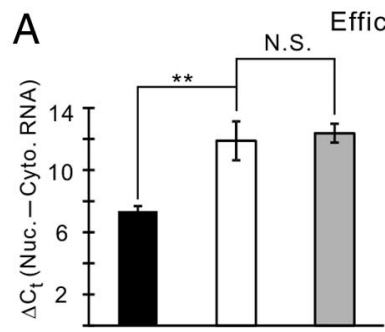

NF-M

Efficiency of Nucleocytoplasmic RNA transport in optic nerve crushed eyes

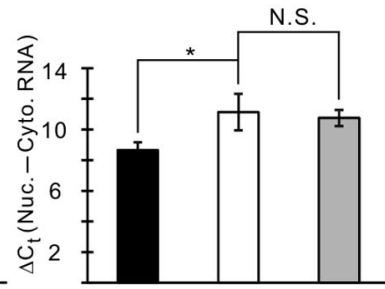

GAP-43

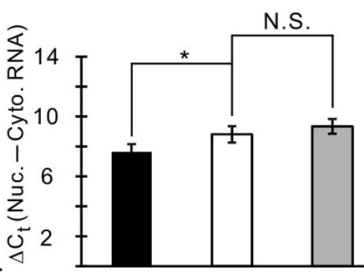

Tau

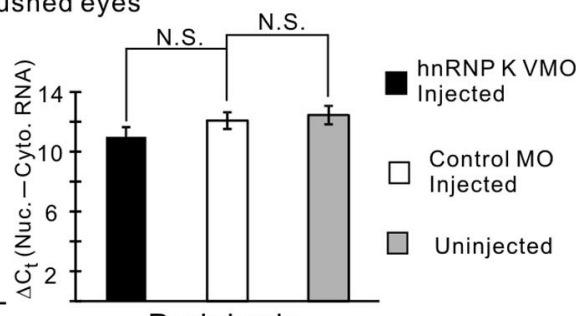

Peripherin

B Polysomal Profiling of NF-M and peripherin in juvenile frog eyes at 12 days post optic nerve crush

hnRNP K VMO inj., Nerve crushed Control MO Inj., Nerve crushed

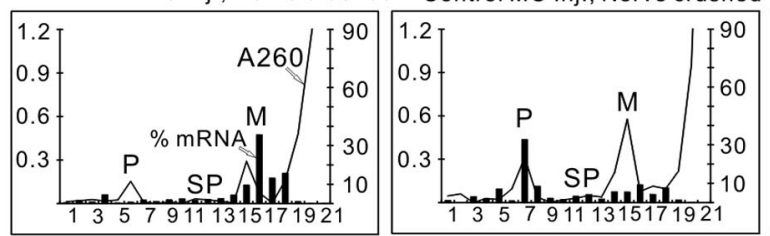

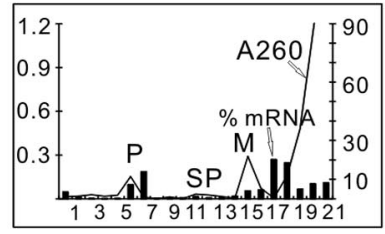

C

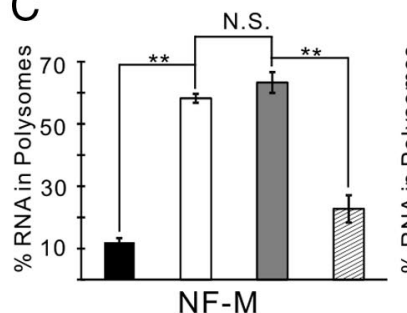

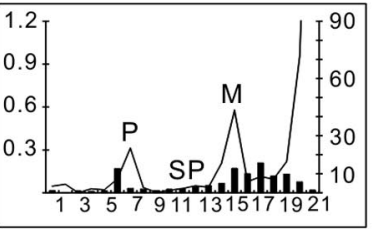

Summary of Translation Profiles for Four Genes

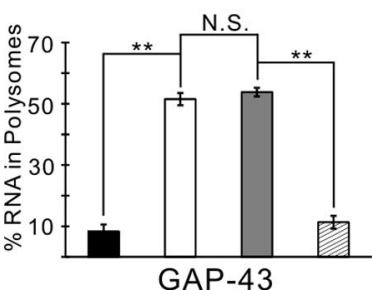

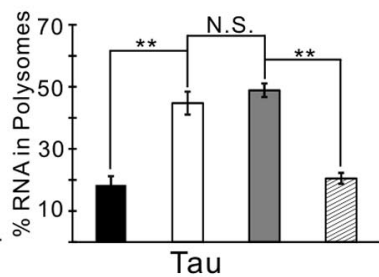

Tau Uninj., Nerve crushed

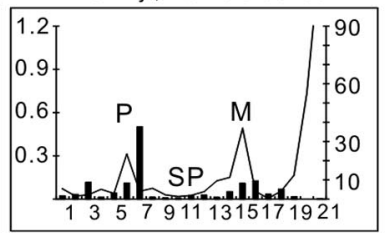

Uninj., Sham operated

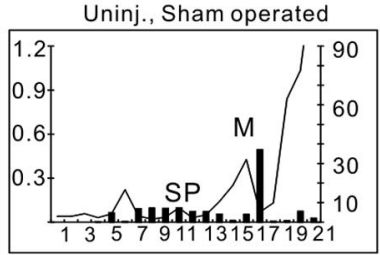

NF-M

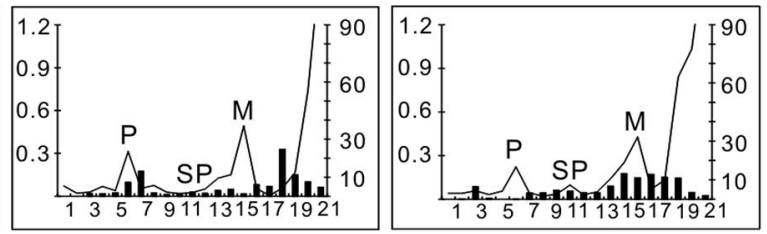

Peripherin

Figure 7. hnRNP K knockdown induces defects in the translation of select cytoskeletal-associated RNA targets during optic axon regeneration. $A$, Efficiency of nuclear export of $N F-M$, GAP-43, tau, and peripherin mRNAs. qRT-PCR of nuclear versus cytosolic fractions from eye was performed for each mRNA at $12 \mathrm{~d}$ after optic nerve crush under the indicated conditions (color key, right). $\Delta C_{t}$, difference in the number of PCR cycles to reach threshold ( \pm SD) between the nuclear and cytosolic fractions $\left({ }^{* *} p \leq 0.01 ;{ }^{*} p \leq 0.05, N\right.$. S., $p>0.25, t$ test on 3 replicates). $\boldsymbol{B}$, Polysomal profiles of NF-M (top) and peripherin (bottom) RNA at $12 \mathrm{~d}$ after optic nerve crush under four different conditions, as indicated. P, polysomal fractions; SP, subpolysomal fractions; M, monosomal fractions; abscissa, fraction number; left ordinate and solid line, total RNA $\left(A_{260}\right)$; right ordinate and bars, percentage of NF-M or peripherin RNA loaded onto the gradient present in each fraction. $C$, The mean percentage ( \pm SD) of each RNA in efficiently translated fractions (percentage RNA in polysomes) of NF-M, GAP-43, tau, and peripherin mRNAs under four different conditions (color key, right). In uninjected eyes, the efficiency of translation increased significantly for all four mRNAs during optic nerve regeneration. This regenerative increase was inhibited by hnRNP K VMO for the three hnRNP K mRNA targets (NF-M, tau, GAP-43) but not for the nontargeted mRNA ( peripherin) ( ${ }^{* *} p \leq 0.01$; N.S., $p>0.15 ; t$ test on 3 replicates).

growth-associated proteins whose RNAs are validated targets of hnRNP K (Irwin et al., 1997; Thyagarajan and Szaro, 2004; Liu and Szaro, 2011). Whereas earlier studies have shown that hnRNP K is required for the initiation of new axons in embryos (Liu et al., 2008; Liu and Szaro, 2011), this study demonstrated for the first time that it is also critical later in life for the regeneration of an axon by mature CNS neurons.

A novel technique was used for achieving targeted geneknockdown of hnRNP $\mathrm{K}$ in RGCs by intravitreal injection of VMOs. VMOs have been used successfully to target gene expression when delivered both intravenously and by local injection into various organs, including the inner ear of chick embryos and the fin and spinal cord of zebrafish (Morcos et al., 2008; Chablais and Jazwinska, 2010; Guo et al., 2011; Kowalik and Hudspeth, 2011). In our case, intravitreal injection proved to be both effective and specific to RGCs, probably because they lie near the retinal surface. The density of the underlying retinal neuropil, coupled with the limited ability of VMOs to cross the blood-brain barrier (Morcos et al., 2008), most likely restricted VMO targeting from deeper retinal cell layers. Thus, the failure of axons to regenerate must have resulted directly from defects intrinsic to the RGCs themselves. Although expression began to recover within a few days of hnRNP K knockdown by a single injection, it was maintained for longer periods by repeated injections, a feature that could be exploited to target gene expression in RGCs within any time window during regeneration. VMOs, therefore, provide a convenient tool for studying gene function in an intact, nontransgenic animal model of successful optic axon regeneration. hnRNP K knockdown stimulated neither increased neurodegeneration beyond that normally caused by optic nerve injury nor any abnormal compensatory RGC proliferation. Their ability to take up WGA, as well as to detect and initiate an injury response, further confirmed that RGCs exposed to VMO were otherwise healthy. Because increased RNA expression resulted in increased protein expression only for those growth-associated mRNAs that were not targeted by hnRNP K (peripherin and $N$ - $\beta$-tubulin), the failure of axons to regenerate must have arisen specifically from defects encompassing hnRNP K's multiple downstream targets. 
In embryos, hnRNP K inhibits axon initiation largely by coregulating multiple RNAs whose proteins organize microfilaments, microtubules, and intermediate filaments into arrangements that are both characteristic of axons and necessary for their initiation and subsequent outgrowth (Liu et al., 2008; Liu and Szaro, 2011). At the molecular level, these targeted RNAs exhibited defects in polysome loading and nuclear export during development. That several of these very same RNAs exhibited similar defects with hnRNP K knockdown during regeneration indicated that the post-transcriptional mechanisms used by hnRNP K to regulate RNA targets mediating axon outgrowth are extensively shared between development and regeneration. Because it was detected in neither embryonic nor RGC axons (Liu et al., 2008), hnRNP K, in both instances, most likely exerted its control via targeting RNAs within the perikaryon, as opposed to the growth cone. Microarray analysis of hnRNP K coimmunoprecipitated RNAs from juvenile Xenopus brain indicates that its targeted RNAs include those of many cytoskeletal-associated proteins [e.g., type IV neurofilaments, GAP-43, tau, actin-related protein 2] as well as ones involved in intracellular transport and trafficking (e.g., KIF3B, RAB5A) (Liu and Szaro, 2011). This, together with the perikaryal accumulation of $\mathrm{N}-\beta$-tubulin in RGCs observed with hnRNP K knockdown, suggests that during regeneration, as in development, the failure of axons to regenerate is in large part caused by downstream defects in the synthesis of proteins that either reorganize the cytoskeleton to initiate regrowth, transport structural materials for sustaining outgrowth, or both.

We were initially surprised that hnRNP K was predominantly nuclear when its effects on translation were greatest. Studies from mammalian systems provide useful insights for interpreting these observations. In HeLa cells, for example, nuclear localization of hnRNP K is associated with increased shuttling of mRNA cargoes to the cytoplasm and is directly coupled with the transcriptional activity of RNA polymerase II (Michael et al., 1997). In neuroblastoma cells, hnRNP K silences translation of $p 21$ until it is displaced from the mRNA (Yano et al., 2005). Together, these studies suggest that hnRNP K enters the nucleus to bind mRNA targets, accompanies them to the cytoplasm while translationally silencing them, and then returns rapidly to the nucleus after releasing its cargo to the translational machinery. Nuclear localization may therefore increase at times when export and translation of RNA targets are greatest, especially if the time spent in the nucleus is the rate-limiting step for shuttling (Michael et al., 1997). Similar phenomena occur with HuR and other RNAbinding proteins that shuttle from the nucleus and have cytoplasmic functions (Nakielny and Dreyfuss, 1997; Fan and Steitz, 1998). These ideas are consistent with our observation that hnRNP K localized to RGC nuclei at times during regeneration (i.e., at 11 but not $3 \mathrm{~d}$ ) when these activities are greatest for NF-M RNA (Ananthakrishnan et al., 2008). These observations place the activation of hnRNP K after injury downstream of the initial injury response, which at $3 \mathrm{~d}$ is marked by dramatic increases in neurofilament gene transcription (Gervasi et al., 2003; Ananthakrishnan et al., 2008; Ananthakrishnan and Szaro, 2009).

hnRNP K thus joins a growing list of molecules crucial for the intrinsic regenerative response of mature CNS neurons (Cho et al., 2005; Moore et al., 2009; Park et al., 2009; Smith et al., 2009; Sun and $\mathrm{He}, 2010)$. Changes in gene transcription have long been known to be important for regeneration, and several transcription factors have been implicated in mediating these changes. The transcription factors $C / E B P \beta$ and vertebrate homologs to Drosophila Krüppel increase in expression early during the regenera- tive response of mouse facial nerve and zebrafish optic nerve, respectively, to promote regeneration and regulate GAP-43 (Nadeau et al., 2005; Kajimura et al., 2007; Veldman et al., 2007). Accumulating evidence points to post-transcriptional regulatory mechanisms as additional key determinants of axon regeneration (Park et al., 2008; Yan et al., 2009; Yang and Yang, 2012). GAP-43 and $N F-M$ are both under strong post-transcriptional control during optic axon regeneration and, in both cases, this control further modulates the primary transcripts to effect downstream changes in cytosolic mRNA levels and translation (PerroneBizzozero et al., 1991; Ananthakrishnan et al., 2008). NF-M transcriptional increases occur in the injured and uninjured eye alike, days before the post-transcriptional events ensue. The latter are restricted to the injured eye in response to cues encountered later by the regenerating axons along the visual pathway (Zhao and Szaro, 1995; Ananthakrishnan et al., 2008). This cascade undoubtedly enables protein supply to better meet demand as it shifts with the dynamics of axon outgrowth.

hnRNP K is ideally situated to mediate the fine-tuning of protein expression triggered by cell-signaling events. It acts as the central RNA-binding scaffolding element of diverse messenger ribonucleoprotein (mRNP) complexes, whose varying compositions direct RNA fates differentially, depending on the cellular context (Bomsztyk et al., 1997; Bomsztyk et al., 2004; Mikula et al., 2006b). Downstream of hnRNP K, $>750$ transcripts from juvenile Xenopus brain have been identified as potential hnRNP $\mathrm{K}$ targets by microarray analysis of coimmunoprecipitated RNAs, including many associated with the cytoskeleton, intracellular trafficking, and eye development, and which could be investigated further for their roles in regeneration (Liu and Szaro, 2011). Upstream, multiple kinases and interacting partners direct the formation of hnRNP K-containing mRNP complexes (Makeyev and Liebhaber, 2002; Bomsztyk et al., 2004; Mikula et al., 2006a,b; Sataranatarajan et al., 2008). They include PKC $\delta$, and Src-family, MAP-family, and JNK-family kinases, all of which have been implicated in axon outgrowth (Ignelzi et al., 1994; Zhao et al., 2003; Ling et al., 2004; Oliva et al., 2006; Nix et al., 2011). This complexity raises the obvious question of whether some feature of the hnRNP K pathway is responsible for the failure of optic axons to regenerate in mammals. Consistent with playing an important role in developing mammalian neurons, hnRNP $\mathrm{K}$ is more widely expressed in the embryonic rat brain than in the mature brain (Blanchette et al., 2006). It seems likely that its role in developing rodent brain is similar to that in axon outgrowth in Xenopus, since rodent and frog hnRNP Ks share extensive sequence identity (Bomsztyk et al., 2004) along with many key RNA targets, including type IV neurofilaments and GAP-43 (Irwin et al., 1997; Thyagarajan and Szaro, 2004, 2008). Future studies of hnRNP K's downstream targets and upstream activators within the RNA regulon active in successful optic axon regeneration, as well as studies of how these features differ with unsuccessful regeneration, should yield key insights into the intrinsic molecular mechanisms underlying CNS axon regeneration.

\section{References}

Ananthakrishnan L, Szaro BG (2009) Transcriptional and translational dynamics of light neurofilament subunit RNAs during Xenopus laevis optic nerve regeneration. Brain Res 1250:27-40.

Ananthakrishnan L, Gervasi C, Szaro BG (2008) Dynamic regulation of middle neurofilament (NF-M) RNA pools during optic nerve regeneration. Neuroscience 153:144-153.

Bates CA, Meyer RL (1993) The heavy neurofilament protein is expressed in regenerating adult but not embryonic mammalian optic fibers in vitro. Exp Neurol 119:249-257. 
Benowitz LI, Shashoua VE, Yoon MG (1981) Specific changes in rapidly transported proteins during regeneration of the goldfish optic nerve. J Neurosci 1:300-307.

Black MM, Slaughter T, Moshiach S, Obrocka M, Fischer I (1996) Tau is enriched on dynamic microtubules in the distal region of growing axons. J Neurosci 16:3601-3619.

Blanchette AR, Fuentes Medel YF, Gardner PD (2006) Cell-type-specific and developmental regulation of heterogeneous nuclear ribonucleoprotein K mRNA in the rat nervous system. Gene Expr Patterns 6:596-606.

Bomsztyk K, Van Seuningen I, Suzuki H, Denisenko O, Ostrowski J (1997) Diverse molecular interactions of the hnRNP K protein. FEBS Lett 403:113-115.

Bomsztyk K, Denisenko O, Ostrowski J (2004) hnRNP K: one protein multiple processes. BioEssays 26:629-638.

Bomze HM, Bulsara KR, Iskandar BJ, Caroni P, Skene JH (2001) Spinal axon regeneration evoked by replacing two growth cone proteins in adult neurons. Nat Neurosci 4:38-43.

Busch A, Hertel KJ (2012) Evolution of SR protein and hnRNP splicing regulatory factors. Wiley Interdiscip Rev RNA 3:1-12.

Chablais F, Jazwinska A (2010) IGF signaling between blastema and wound epidermis is required for fin regeneration. Development 137:871-879.

Chidlow G, Wood JP, Sarvestani G, Manavis J, Casson RJ (2009) Evaluation of Fluoro-Jade $\mathrm{C}$ as a marker of degenerating neurons in the rat retina and optic nerve. Exp Eye Res 88:426-437.

Cho KS, Yang L, Lu B, Feng Ma H, Huang X, Pekny M, Chen DF (2005) Re-establishing the regenerative potential of central nervous system axons in postnatal mice. J Cell Sci 118:863-872.

Fan XC, Steitz JA (1998) Overexpression of HuR, a nuclear-cytoplasmic shuttling protein, increases the in vivo stability of ARE-containing mRNAs. EMBO J 17:3448-3460.

Fawcett JW, Gaze RM (1981) The organization of regenerating axons in the Xenopus optic nerve. Brain Res 229:487-490.

Filbin MT (2006) Recapitulate development to promote axonal regeneration: good or bad approach? Philos Trans R Soc Lond B Biol Sci 361:1565-1574.

Gaze RM (1959) Regeneration of the optic nerve in Xenopus laevis. Q J Exp Physiol Cogn Med Sci 44:290-308.

Gervasi C, Stewart CB, Szaro BG (2000) Xenopus laevis peripherin (XIF3) is expressed in radial glia and proliferating neural epithelial cells as well as in neurons. J Comp Neurol 423:512-531.

Gervasi C, Thyagarajan A, Szaro BG (2003) Increased expression of multiple neurofilament mRNAs during regeneration of vertebrate central nervous system axons. J Comp Neurol 461:262-275.

Gibbs KM, Chittur SV, Szaro BG (2011) Metamorphosis and the regenerative capacity of spinal cord axons in Xenopus laevis. Eur J Neurosci 33:9-25.

Glasgow E, Druger RK, Levine EM, Fuchs C, Schechter N (1992) Plasticin, a novel type III neurofilament protein from goldfish retina: increased expression during optic nerve regeneration. Neuron 9:373-381.

Goodbrand IA, Gaze RM (1991) Microglia in tadpoles of Xenopus laevis: normal distribution and the response to optic nerve injury. Anat Embryol 184:71-82.

Gravina P, Campioni N, Loreni F, Pierandrei-Amaldi P, Cardinali B (2002) Complementary DNA analysis, expression and subcellular localization of hnRNP E2 gene in Xenopus laevis. Gene 290:193-201.

Guo Y, Ma L, Cristofanilli M, Hart RP, Hao A, Schachner M (2011) Transcription factor Sox $11 \mathrm{~b}$ is involved in spinal cord regeneration in adult zebrafish. Neurosci 172:329-341.

Heacock AM, Agranoff BW (1976) Enhanced labeling of a retinal protein during regeneration of optic nerve in goldfish. Proc Natl Acad Sci U S A 73:828-832.

Ignelzi MA Jr, Miller DR, Soriano P, Maness PF (1994) Impaired neurite outgrowth of src-minus cerebellar neurons on the cell adhesion molecule L1. Neuron 12:873-884.

Irwin N, Baekelandt V, Goritchenko L, Benowitz LI (1997) Identification of two proteins that bind to a pyrimidine-rich sequence in the $3^{\prime}$ untranslated region of GAP-43 mRNA. Nucleic Acids Res 25:1281-1288.

Jacobson M (1961) The recovery of electrical activity in the optic tectum of the frog during early regeneration of the optic nerve. Proc R Soc Edin Sect B 28:131-137.

Kajimura D, Dragomir C, Ramirez F, Laub F (2007) Identification of genes regulated by transcription factor KLF7 in differentiating olfactory sensory neurons. Gene 388:34-42.

Kaneda M, Nagashima M, Nunome T, Muramatsu T, Yamada Y, Kubo M, Muramoto K, Matsukawa T, Koriyama Y, Sugitani K, Vachkov IH, Mawatari K, Kato S (2008) Changes of phospho-growth-associated protein 43 (phospho-GAP-43) in the zebrafish retina after optic nerve injury: a long term observation. Neurosci Res 61:281-288.

Kowalik L, Hudspeth AJ (2011) A search for factors specifying tonotopy implicates DNER in hair-cell development in the chick's cochlea. Dev Biol 354:221-231.

Kruger K, Tam AS, Lu C, Sretavan DW (1998) Retinal ganglion cell axon progression from the optic chiasm to initiate optic tract development requires cell autonomous function of GAP-43. J Neurosci 18:5692-5705.

Kusik BW, Hammond DR, Udvadia AJ (2010) Transcriptional regulatory regions of gap43 needed in developing and regenerating retinal ganglion cells. Dev Dyn 239:482-495.

Leibovici M, Monod G, Géraudie J, Bravo R, Méchali M (1992) Nuclear distribution of PCNA during embryonic development in Xenopus laevis: a reinvestigation of early cell cycles. J Cell Sci 102:63-69.

Leu B, Koch E, Schmidt JT (2010) GAP43 phosphorylation is critical for growth and branching of retinotectal arbors in zebrafish. Dev Neurobiol 70:897-911.

Lin W, Szaro BG (1995) Neurofilaments help maintain normal morphologies and support elongation of neurites in Xenopus laevis cultured embryonic spinal cord neurons. J Neurosci 15:8331-8344.

Ling M, Trollér U, Zeidman R, Lundberg C, Larsson C (2004) Induction of neurites by the regulatory domains of PKCdelta and epsilon is counteracted by PKC catalytic activity and the RhoA pathway. Exp Cell Res 292:135-150.

Liu Y, Szaro BG (2011) hnRNP K post-transcriptionally co-regulates multiple cytoskeletal genes needed for axonogenesis. Development 138:3079-3090

Liu Y, Gervasi C, Szaro BG (2008) A crucial role for hnRNP K in axon development in Xenopus laevis. Development 135:3125-3135.

Makeyev AV, Liebhaber SA (2002) The poly(C)-binding proteins: a multiplicity of functions and a search for mechanisms. RNA 8:265-278.

Michael WM, Eder PS, Dreyfuss G (1997) The K nuclear shuttling domain: a novel signal for nuclear import and nuclear export in the hnRNP K protein. EMBO J 16:3587-3598.

Mikucki SA, Oblinger MM (1991) Corticospinal neurons exhibit a novel pattern of cytoskeletal gene expression after injury. J Neurosci Res 30:213-225.

Mikula M, Karczmarski J, Dzwonek A, Rubel T, Hennig E, Dadlez M, Bujnicki JM, Bomsztyk K, Ostrowski J (2006a) Casein kinases phosphorylate multiple residues spanning the entire hnRNP K length. Biochim Biophys Acta 1764:299-306.

Mikula M, Dzwonek A, Karczmarkski J, Rubel T, Dadlez M, Wyrwicz LS, Bomsztyk K, Ostrowski J (2006b) Landscape of the hnRNP K proteinprotein interactome. Proteomics 6:2395-2406.

Moody SA, Miller V, Spanos A, Frankfurter A (1996) Developmental expression of a neuron-specific beta-tubulin in frog (Xenopus laevis): a marker for growing axons during the embryonic period. J Comp Neurol 364:219-230.

Moore DL, Blackmore MG, Hu Y, Kaestner KH, Bixby JL, Lemmon VP, Goldberg JL (2009) KLF family members regulate intrinsic axon regeneration ability. Science 326:298-301.

Morcos PA, Li Y, Jiang S (2008) Vivo-Morpholinos: a non-peptide transporter delivers morpholinos into a wide array of mouse tissues. Biotechniques 45:613-614, 616, 618 passim.

Nadeau S, Hein P, Fernandes KJ, Peterson AC, Miller FD (2005) A transcriptional role for C/EBP $\beta$ in the neuronal response to axonal injury. Mol Cell Neurosci 29:525-535.

Nakielny S, Dreyfuss G (1997) Nuclear export of proteins and RNAs. Curr Opin Cell Biol 9:420-429.

Nix P, Hisamoto N, Matsumoto K, Bastiani M (2011) Axon regeneration requires coordinate activation of p38 and JNK MAPK pathways. Proc Natl Acad Sci U S A 108:10738-10743.

Oliva AA Jr, Atkins CM, Copenagle L, Banker GA (2006) Activated c-Jun $\mathrm{N}$-terminal kinase is required for axon formation. J Neurosci 26:9462-9470.

Ostareck-Lederer A, Ostareck DH, Hentze MW (1998) Cytoplasmic regu- 
latory functions of the KH domain proteins hnRNPs K and E1/E2. Trends Biochem Sci 23:409-411.

Park KK, Liu K, Hu Y, Smith PD, Wang C, Cai B, Xu B, Connolly L, Kramvis I, Sahin M, He Z (2008) Promoting axon regeneration in the adult CNS by modulation of the PTEN/mTOR pathway. Science 322:963-966.

Park KK, Hu Y, Muhling J, Pollett MA, Dallimore EJ, Turnley AM, Cui Q, Harvey AR (2009) Cytokine-induced SOCS expression is inhibited by cAMP analogue: impact on regeneration in injured retina. Mol Cell Neurosci 41:313-324.

Park KK, Liu K, Hu Y, Kanter JL, He Z (2010) PTEN/mTOR and axon regeneration. Exp Neurol 223:45-50.

Pearson ES, Stephens MA (1964) The ratio of range to standard deviation in the same normal sample distribution. Biometrika 51:484-487.

Perrone-Bizzozero NI, Neve RL, Irwin N, Lewis S, Fischer I, Benowitz LI (1991) Post-transcriptional regulation of GAP-43 mRNA levels during neuronal differentiation and nerve regeneration. Mol Cell Neurosci 2:402-409.

Rodger J, Bartlett CA, Harman AM, Thomas C, Beazley LD, Dunlop SA (2001) Evidence that regenerating optic axons maintain long-term growth in the lizard Ctenophorus ornatus: growth-associated protein-43 and gefiltin expression. Neurosci 102:647-654.

Sachs L (1984) Comparison of an empirical distribution with the normal distribution. In: Applied statistics: a handbook of techniques (Reynarowych Z, ed), pp 322-329. New York: Springer.

Sataranatarajan K, Lee MJ, Mariappan MM, Feliers D (2008) PKC-delta regulates the stimulation of vascular endothelial factor mRNA translation by angiotensin II through hnRNP K. Cell Signal 20:969-977.

Schaden H, Stuermer CA, Bähr M (1994) GAP-43 immunoreactivity and axon regeneration in retinal ganglion cells of the rat. J Neurobiol 25:1570-1578.

Schmued LC, Stowers CC, Scallet AC, Xu L (2005) Fluoro-Jade C results in ultra high resolution and contrast labeling of degenerating neurons. Brain Res 1035:24-31.

Schrama LH, Lepperdinger G, Moritz A, van den Engel NK, Marquart A, Oestreicher AB, Eggen BJ, Hage WJ, Richter K, Destrée OHJ (1997) B-50/growth-associated protein-43, a marker of neural development in Xenopus laevis. Neuroscience 76:635-652.

Skene JH, Willard M (1981) Changes in axonally transported proteins during axon regeneration in toad retinal ganglion cells. J Cell Biol 89:86-95.

Smith PD, Sun F, Park KK, Cai B, Wang C, Kuwako K, Martinez-Carrasco I, Connolly L, He Z (2009) SOCS3 deletion promotes optic nerve regeneration in vivo. Neuron 64:617-623.

Sperry RW (1944) Optic nerve regeneration with return of vision in anurans. J Neurophysiol 7:351-361.

Sperry RW (1948) Patterning of central synapses in regeneration of the optic nerve in teleosts. Physiol Zool 21:351-361.

Strittmatter SM, Fankhauser C, Huang PL, Mashimo H, Fishman MC (1995) Neuronal pathfinding is abnormal in mice lacking the neuronal growth cone protein GAP-43. Cell 80:445-452.

Sun F, He Z (2010) Neuronal intrinsic barriers for axon regeneration in the adult CNS. Curr Opin Neurobiol 20:510-518.

Szaro BG, Loh YP, Hunt RK (1985) Specific changes in axonally transported proteins during regeneration of the frog (Xenopus laevis) optic nerve. J Neurosci 5:192-208.

Szaro BG, Lee VMY, Gainer H (1989) Spatial and temporal expression of phosphorylated and non-phosphorylated forms of neurofilament pro- teins in the developing nervous system of Xenopus laevis. Dev Brain Res 48:87-103.

Tanaka EM, Ferretti P (2009) Considering the evolution of regeneration in the central nervous system. Nat Rev Neurosci 10:713-723.

Taylor JS, Jack JL, Easter SS Jr (1989) Is the capacity for optic nerve regeneration related to continued retinal ganglion cell production in the frog? A test of the hypothesis that neurogenesis and axon regeneration are obligatorily linked. Eur J Neurosci 1:626-638.

Thyagarajan A, Szaro BG (2004) Phylogenetically conserved binding of specific $\mathrm{KH}$ domain proteins to the $3^{\prime}$ untranslated region of the vertebrate middle neurofilament mRNA. J Biol Chem 279:49680-49688.

Thyagarajan A, Szaro BG (2008) Dynamic endogenous association of neurofilament mRNAs with K-homology domain ribonucleoproteins in developing cerebral cortex. Brain Res 1189:33-42.

Udvadia AJ, Köster RW, Skene JH (2001) GAP-43 promoter elements in transgenic zebrafish reveal a difference in signals for axon growth during CNS development and regeneration. Development 128:1175-1182.

Veldman MB, Bemben MA, Thompson RC, Goldman D (2007) Gene expression analysis of zebrafish retinal ganglion cells during optic nerve regeneration identifies KLF6a and KLF7a as important regulators of axon regeneration. Dev Biol 312:596-612.

Walker KL, Yoo HK, Undamatla J, Szaro BG (2001) Loss of neurofilaments alters axonal growth dynamics. J Neurosci 21:9655-9666.

Waseem NH, Lane DP (1990) Monoclonal antibody analysis of the proliferating cell nuclear antigen (PCNA). J Cell Sci 96:121-129.

Wetzel DM, Lee VM, Erulkar SD (1989) Long term cultures of neurons from adult frog brain express GABA and glutamate-activated channels. J Neurobiol 20:255-270.

Wilson MA, Gaze RM, Goodbrand IA, Taylor JSH (1992) Regeneration in the Xenopus laevis tadpole optic nerve is preceded by a massive macrophage/microglial response. Anat Embryol 186:75-89.

Yan D, Wu Z, Chisholm AD, Jin Y (2009) The DLK-1 kinase promotes mRNA stability and local translation in C. elegans synapses and axon regeneration. Cell 138:1005-1018.

Yang P, Yang Z (2012) Enhancing intrinsic growth capacity promotes adult CNS regeneration. J Neurol Sci 312:1-6.

Yano M, Okano HJ, Okano H (2005) Involvement of Hu and heterogeneous nuclear ribonucleoprotein $\mathrm{K}$ in neuronal differentiation through $\mathrm{p} 21$ mRNA post-transcriptional regulation. J Biol Chem 280:12690-12699.

Zhang Y, Bo X, Schoepfer R, Holtmaat AJ, Verhaagen J, Emson PC, Lieberman AR, Anderson PN (2005) Growth-associated protein GAP-43 and L1 act synergistically to promote regenerative growth of Purkinje cell axons in vivo. Proc Natl Acad Sci U S A 102:14883-14888.

Zhao Y, Szaro BG (1994) The return of phosphorylated and nonphosphorylated epitopes of neurofilament proteins to the regenerating optic nerve of Xenopus laevis. J Comp Neurol 343:158-172.

Zhao Y, Szaro BG (1995) The optic tract and tectal ablation influence the composition of neurofilaments in regenerating optic axons of Xenopus laevis. J Neurosci 15:4629-4640.

Zhao YL, Takagawa K, Oya T, Yang HF, Gao ZY, Kawaguchi M, Ishii Y, Sasaoka T, Owada K, Furuta I, Sasahara M (2003) Active Src expression is induced after rat peripheral nerve injury. Glia 42:184-193.

Zhu Q, Couillard-Després S, Julien JP (1997) Delayed maturation of regenerating myelinated axons in mice lacking neurofilaments. Exp Neurol 148:299-316 